\title{
STABLE PAIR INVARIANTS OF SURFACES AND SEIBERG-WITTEN INVARIANTS
}

\author{
MARTIJN KOOL
}

\begin{abstract}
The moduli space of stable pairs on a local surface $X=K_{S}$ is in general non-compact. The action of $\mathbb{C}^{*}$ on the fibres of $X$ induces an action on the moduli space and the stable pair invariants of $X$ are defined by the virtual localization formula. We study the contribution to these invariants of stable pairs (scheme theoretically) supported in the zero section $S \subset X$. Sometimes there are no other contributions, e.g. when the curve class $\beta$ is irreducible.

We relate these surface stable pair invariants to the Poincaré invariants of Dürr-Kabanov-Okonek. The latter are equal to the Seiberg-Witten invariants of $S$ by work of Dürr-Kabanov-Okonek and Chang-Kiem. We give two applications of our result. (1) For irreducible curve classes the GW/PT correspondence for $X=K_{S}$ implies Taubes' GW/SW correspondence for $S$. (2) When $p_{g}(S)=0$, the difference of surface stable pair invariants in class $\beta$ and $K_{S}-\beta$ is a universal topological expression.
\end{abstract}

\section{INTRODUCTION}

In 23, R. Pandharipande and R. P. Thomas introduce stable pairs on projective 3 -folds $X$ and show their moduli space is a component of the moduli space of all complexes in the bounded derived category $D^{b}(X)$. Formally, a stable pair $(F, s)$ on $X$ consists of a pure dimension 1 sheaf $F$ on $X$ and a section $s \in H^{0}(F)$ with 0 -dimensional cokernel. The moduli space of stable pairs has a perfect obstruction theory, which is symmetric in the case $X$ is Calabi-Yau. The associated invariants are known as stable pair invariants and are closely related to the Donaldson-Thomas and Gromov-Witten invariants of $X[3,16,17,18,21,22,23,24,31$.

We consider the case where $X=K_{S}$ is the total space of the canonical bundle over a smooth projective surface $S$. Let $P_{\chi}(X, \beta)$ denote the moduli space of stable pairs $(F, s)$ on $X$ with class $\beta \in H_{2}(S)$ and $\chi(F)=\chi$. The space $P_{\chi}(X, \beta)$ carries a perfect obstruction theory but can be non-compact. Using the $\mathbb{C}^{*}$-action on the fibres of $X$ gives an induced obstruction theory on $P_{\chi}(X, \beta)^{\mathbb{C}^{*}}$. The components of this fixed locus are compact. For any

\footnotetext{
${ }^{1}$ We denote the $\mathbb{C}^{*}$-equivariant cohomology of $X$ by $H_{\mathbb{C}^{*}}^{*}(X, \mathbb{Q})$. Endowing $S$ with trivial $\mathbb{C}^{*}$-action, we then have $H_{\mathbb{C}^{*}}^{*}(X, \mathbb{Q}) \cong H_{\mathbb{C}^{*}}^{*}(S, \mathbb{Q})$.
} 
$\sigma_{1}, \ldots, \sigma_{m} \in H_{\mathbb{C}^{*}}^{*}(S, \mathbb{Q})$ the stable pair invariants of $X$ are defined by the virtual localization formula of T. Graber and R. Pandharipande [12]:

$$
P_{\chi, \beta}\left(X, \tau_{\alpha_{1}}\left(\sigma_{1}\right) \cdots \tau_{\alpha_{m}}\left(\sigma_{m}\right)\right):=\int_{\left[P_{\chi}(X, \beta)^{\mathbb{C}^{*}}\right]^{\operatorname{vir}}} \frac{1}{e\left(N^{\text {vir }}\right)} \prod_{i=1}^{m} \tau_{\alpha_{i}}\left(\sigma_{i}\right) .
$$

Here $e\left(N^{\mathrm{vir}}\right)$ is the equivariant Euler class of the virtual normal bundle and $\tau_{\alpha}(\sigma)$ is the descendent insertion

$$
\tau_{\alpha}(\sigma):=\pi_{P *}\left(\pi_{X}^{*}(\sigma) \cap \operatorname{ch}_{\alpha+2}^{\mathbb{C}^{*}}(\mathbb{F})\right)
$$

where $\alpha_{i} \geq 0, \mathbb{F}$ is the universal sheaf on $P_{\chi}(X, \beta) \times X$, and $\operatorname{ch}^{\mathbb{C}^{*}}$ denotes $\mathbb{C}^{*}$-equivariant Chern character. Note that these invariants are elements of $\mathbb{Q}\left[t, t^{-1}\right]$, where $t$ is the equivariant parameter. In this paper we will only be concerned with primary point insertions

$$
\tau_{0}(\mathrm{pt}):=\pi_{P *}\left(\pi_{X}^{*}(\mathrm{pt}) \cap \mathrm{ch}_{2}^{\mathbb{C}^{*}}(\mathbb{F})\right)
$$

where pt denotes the (Poincaré dual of) the point class in $H^{4}(S, \mathbb{Z})$.

The easiest component of $P_{\chi}(X, \beta)^{\mathbb{C}^{*}}$ consists of stable pairs which are scheme theoretically supported on the zero section $S \subset X$, i.e. $P_{\chi}(S, \beta)$. Denote the Hilbert scheme of effective divisors on $S$ with class $\beta$ by $H_{\beta}:=\operatorname{Hilb}_{\beta}(S)$ and the universal curve by $\mathcal{C} \rightarrow H_{\beta}$. Let $n$ be determined by $\chi=1-h+n$, where $h$ is the arithmetic genus of curves with class $\beta$

$$
2 h-2=\beta(\beta+\mathrm{k}), \mathbf{k}:=c_{1}\left(\mathcal{O}\left(K_{S}\right)\right) \in H^{2}(S, \mathbb{Z}) .
$$

Given a stable pair $\left[s: \mathcal{O}_{S} \rightarrow F\right]$ on $S$, the scheme theoretic support of $F$ is a Gorenstein curve $C \subset S$. The cokernel $Q$ of $s$ gives rise to a 0 -dimensional closed subscheme $Z \subset C$ via the surjection $\mathcal{O}_{C} \rightarrow \mathscr{E} x t^{1}\left(Q, \mathcal{O}_{C}\right)$ obtained by dualizing. This provides an isomorphism [25]

$$
P_{\chi}(S, \beta) \cong \operatorname{Hilb}^{n}\left(\mathcal{C} / H_{\beta}\right),
$$

where $\operatorname{Hilb}^{n}\left(\mathcal{C} / H_{\beta}\right)$ is the relative Hilbert scheme of $n$ points on the fibres of $\mathcal{C} \rightarrow H_{\beta}$. In this paper we only consider contributions to (11) of the "surface component" $P_{\chi}(S, \beta)$, i.e.

$$
P_{\chi, \beta}\left(S, \tau_{\alpha_{1}}\left(\sigma_{1}\right) \cdots \tau_{\alpha_{m}}\left(\sigma_{m}\right)\right):=\int_{\left[P_{\chi}(S, \beta)\right] \operatorname{vir}} \frac{1}{e\left(N^{\text {vir }}\right)} \prod_{i=1}^{m} \tau_{\alpha_{i}}\left(\sigma_{i}\right) .
$$

We group these invariants together into a generating function

$$
\mathbf{Z}_{\beta}^{P}\left(S, \tau_{\alpha_{1}}\left(\sigma_{1}\right) \cdots \tau_{\alpha_{m}}\left(\sigma_{m}\right)\right):=\sum_{\chi \in \mathbb{Z}} P_{\chi, \beta}\left(S, \tau_{\alpha_{1}}\left(\sigma_{1}\right) \cdots \tau_{\alpha_{m}}\left(\sigma_{m}\right)\right) q^{\chi} .
$$

The following is our main theorem: 
Theorem 1.1. For any $S, \beta$, and $m:=\frac{\beta(\beta-\mathrm{k})}{2}$

$$
\mathrm{Z}_{\beta}^{P}\left(S, \tau_{0}(\mathrm{pt})^{m}\right)=t^{m} P_{S}(\beta)\left(q^{\frac{1}{2}}+q^{-\frac{1}{2}}\right)^{2 h-2},
$$

where $t$ is the equivariant parameter, $2 h-2=\beta(\beta+\mathrm{k})$, and $P_{S}(\beta) \in \mathbb{Z}$ is the numerical part of the Poincaré invariant $P_{S}^{+}(\beta)$ of Dürr-Kabanov-Okonek.

In this theorem

$$
P_{S}^{+}(\beta) \in \Lambda^{*} H^{1}(S, \mathbb{Z})^{*}
$$

are the Poincaré invariants of $S, \beta$ defined by M. Dürr, A. Kabanov, and Ch. Okonek [6]. These invariants are defined in terms of a natural virtual cycle on the Hilbert scheme of curves $H_{\beta}$. They define a corresponding invariant $P_{S}^{-}(\beta)$ in terms of a natural virtual cycle on $H_{\mathrm{k}-\beta}$. We are only concerned with the numerical part (degree $b_{1}(S)$ in cohomology), which we denote by $P_{S}(\beta)$. Dürr-Kabanov-Okonek conjectured that $P_{S}^{ \pm}(\beta)$ are equal to the Seiberg-Witten invariants of $S, \beta$. Up to a purely algebraic conjecture, they prove this using their wall-crossing and blow-up formula. This algebraic conjecture was subsequently proved by H.-l. Chang and Y.-H. Kiem via a beautiful application of cosection localization [4]. As a corollary of the "Poincaré/PT correspondence" of Theorem 1.1 and the (much deeper!) Poincaré/SW correspondence of [4, 6] we obtain:

Corollary 1.2. In the notation of Theorem 1.1

$$
\mathrm{Z}_{\beta}^{P}\left(S, \tau_{0}(\mathrm{pt})^{m}\right)=t^{m} S W(\beta)\left(q^{\frac{1}{2}}+q^{-\frac{1}{2}}\right)^{2 h-2},
$$

where $S W(\beta) \in \mathbb{Z}$ is the Seiberg-Witten invariant of $S, \beta$.

We have two applications of Theorem 1.1 (and its Corollary 1.2). The first is to Gromov-Witten theory. For any $g$, let $\bar{M}_{g, m}^{\prime}(X, \beta)$ be the moduli space of stable maps with possibly disconnected domain curve and no collapsed connected components. Its $\mathbb{C}^{*}$-fixed locus $\bar{M}_{g, m}^{\prime}(S, \beta)$ has an induced perfect obstruction theory, which is the usual Gromov-Witten theory of $S$. The GromovWitten invariants of $X$ are defined by virtual localization

$$
\begin{aligned}
& R_{g, \beta}\left(X, \tau_{\alpha_{1}}\left(\sigma_{1}\right) \cdots \tau_{\alpha_{m}}\left(\sigma_{m}\right)\right):=\int_{\left.\bar{M}_{g, m}^{\prime}(S, \beta)\right]^{\mathrm{vir}}} \frac{1}{e\left(N^{\mathrm{vir}}\right)} \prod_{i=1}^{m} \tau_{\alpha_{i}}\left(\sigma_{i}\right), \\
& \tau_{\alpha_{i}}\left(\sigma_{i}\right):=\psi_{i}^{\alpha_{i}} \operatorname{ev}_{i}^{*}\left(\sigma_{i}\right), \\
& \mathrm{Z}_{\beta}^{G W}\left(X, \tau_{\alpha_{1}}\left(\sigma_{1}\right) \cdots \tau_{\alpha_{m}}\left(\sigma_{m}\right)\right):=\sum_{g} R_{g, \beta}\left(X, \tau_{\alpha_{1}}\left(\sigma_{1}\right) \cdots \tau_{\alpha_{m}}\left(\sigma_{m}\right)\right) u^{2 g-2},
\end{aligned}
$$

where $\psi_{i}$ are the $\psi$-classes and $\mathrm{ev}_{i}$ the evaluation maps. From Theorem 1.1 (or rather Corollary 1.2) we will deduce: 
Theorem 1.3. Fix any $S, \beta$ with $\beta$ irreducible. Let $m:=\frac{\beta(\beta-\mathrm{k})}{2}$ and $2 h-2=$ $\beta(\beta+\mathrm{k})$. The $G W / P T$ correspondence 2 for $\mathbf{Z}_{\beta}^{G W}\left(X, \tau_{0}(\mathrm{pt})^{m}\right)$ and $\mathbf{Z}_{\beta}^{P}\left(X, \tau_{0}(\mathrm{pt})^{m}\right)$ is equivalent to the following equality

$$
\mathrm{Z}_{\beta}^{G W}\left(X, \tau_{0}(\mathrm{pt})^{m}\right)=t^{m} S W(\beta)(2 \sin (u / 2))^{2 h-2} .
$$

In particular, setting $-q=e^{i u}$, the lowest order terms of $Z_{\beta}^{G W}\left(X, \tau_{0}(\mathrm{pt})^{m}\right)$ and $\mathrm{Z}_{\beta}^{P}\left(X, \tau_{0}(\mathrm{pt})^{m}\right)$ in $u$ coincide if and only if

$$
S W(\beta)=\int_{\left[\bar{M}_{h, m}^{\prime}(S, \beta)\right]^{\mathrm{vir}}} \prod_{i=1}^{m} \tau_{0}(\mathrm{pt}) .
$$

We have a similar result for any $S, \beta$ with $-K_{S}$ nef and $\beta$ sufficiently ample (Remark 2.3). This shows that the GW/PT correspondence implies (a very special case of) Taubes' GW/SW correspondence [29, 30].

The second application of Theorem 1.1 is a universal formula for the difference of stable pair invariants in class $\beta$ and $k-\beta$. Instead of the stable pair invariants (3), one can define reduced stable pair invariants of $X$ in class $\beta$

$$
P_{\chi, \beta}^{\mathrm{red}}\left(X, \tau_{\alpha_{1}}\left(\sigma_{1}\right) \cdots \tau_{\alpha_{m}}\left(\sigma_{m}\right)\right)
$$

These originate from stable pair theory on $P_{\chi}(X, \beta)$ by removing a trivial part of rank $p_{g}(S):=h^{0,2}(S)$ from the obstruction bundle. The reduced invariants coincide with the usual invariants when $p_{g}(S)=0$. Reduced stable pair invariants have been studied by many people: see [14] and references therein. Consider the surface part of these invariants for any number of point insertions 3

$$
P_{\chi, \beta}^{\mathrm{red}}\left(S, \tau_{0}(\mathrm{pt})^{m}\right) \text {. }
$$

We recall the definition in Appendix $\mathrm{A}$, where we give a formula for the reduced virtual cycle (Proposition A.1). This formula is not used in the main body of this text, but is of independent interest. It extends a formula from [15, Appendix], which was derived under the following condition

$$
H^{2}(L)=0 \text { for all line bundles } L \text { with } c_{1}(L)=\beta \text {. }
$$

When Condition (44) is satisfied it is shown in [15] that $P_{\chi, \beta}^{\mathrm{red}}\left(S, \tau_{0}(\mathrm{pt})^{m}\right)$ is given by a universal function in $\beta^{2}, \beta . c_{1}(S), c_{1}(S)^{2}, c_{2}(S)$, and certain invariants of the ring structure of $H^{*}(S, \mathbb{Z})$. The precise statement is recalled in Theorem A.2 of Appendix A. It is natural to ask whether universality holds for all invariants $P_{\chi, \beta}^{\mathrm{red}}\left(S, \tau_{0}(\mathrm{pt})^{m}\right), P_{\chi, \beta}\left(S, \tau_{0}(\mathrm{pt})^{m}\right)$. We show that this is not the case

\footnotetext{
2.e. [23, Conj. 3.3] but for $X$ a non-compact Calabi-Yau 3-fold. See also [19, Sect. 1.4].

${ }^{3}$ So $m$ need not be $\frac{\beta(\beta-\mathrm{k})}{2}$ as in Theorems 1.1 and 1.3 .

${ }^{4}$ This universality result is used in the recent proof of the Katz-Klemm-Vafa conjecture for all curve classes by R. Pandharipande and R. P. Thomas [26].
} 
(Remark A.3 $)$. The reason is as follows. Theorem 1.1 relates $P_{\chi, \beta}\left(S, \tau_{0}(\mathrm{pt})^{m}\right)$ to Poincaré invariants. Using examples of [6] we observe that Poincaré invariants do not satisfy universality (Examples B.1, B.3, B.5 of Appendix B).

Despite failure of universality there is an interesting "duality" for surfaces with $p_{g}(S)=0$. If $\beta$ or $\mathrm{k}-\beta$ satisfies Condition (44), then one of

$$
P_{\chi, \beta}\left(S, \tau_{0}(\mathrm{pt})^{m}\right), P_{\chi, \mathrm{k}-\beta}\left(S, \tau_{0}(\mathrm{pt})^{m}\right)
$$

is given by a universal expression and the other is zero. These cases are covered by [15]. The new case is when neither $\beta$ nor $\mathrm{k}-\beta$ satisfies (4). Then universality can fail for the individual invariants $P_{\chi, \beta}\left(S, \tau_{0}(\mathrm{pt})^{m}\right), P_{\chi, \mathrm{k}-\beta}\left(S, \tau_{0}(\mathrm{pt})^{m}\right)$ (Examples B.1, B.3, B.5 of Appendix B), but their difference satisfies a nice duality formula. Combining Theorem 1.1 and the wall-crossing formula of Dürr-Kabanov-Okonek will lead to the following theorem:

Theorem 1.4. Fix $S, \beta$ such that $p_{g}(S)=0$ and neither $\beta$ nor $\mathrm{k}-\beta$ satisfies Condition (4)). If $\beta(\beta-\mathbf{k})<0$, then

$$
\begin{gathered}
P_{\chi, \beta}\left(S, \tau_{0}(\mathrm{pt})^{m}\right)=P_{\chi, \mathrm{k}-\beta}\left(S, \tau_{0}(\mathrm{pt})^{m}\right)=0 . \\
\text { If } \beta(\beta-\mathrm{k}) \geq 0 \text {, then } \beta(\beta-\mathrm{k})=0, q(S):=h^{0,1}(S)=1 \text {, and } \\
P_{\chi, \beta}\left(S, \tau_{0}(\mathrm{pt})^{m}\right)=P_{\chi, \mathrm{k}-\beta}\left(S, \tau_{0}(\mathrm{pt})^{m}\right)=0, \text { for } m>0 \\
\frac{\mathrm{Z}_{\beta}^{P}(S)}{\left(q^{\frac{1}{2}}+q^{-\frac{1}{2}}\right)^{2 \beta^{2}}}-\frac{\mathrm{Z}_{\mathrm{k}-\beta}^{P}(S)}{\left(q^{\frac{1}{2}}+q^{-\frac{1}{2}}\right)^{2(\mathrm{k}-\beta)^{2}}}=\frac{1}{2}[\beta]-\frac{1}{2}[\mathrm{k}-\beta] \text {, for } m=0 .
\end{gathered}
$$

Examples of $S, \beta$ with $p_{g}(S)=0, \beta(\beta-\mathrm{k}) \geq 0$, and neither $\beta$ nor $\mathrm{k}-\beta$ satisfying Condition (44) are given in Remark B.7 of Appendix B. Such surfaces are necessarily elliptic fibrations or blow-ups thereof. The results of this paper make heavy use of the work of Dürr-Kabanov-Okonek [6]. For the purposes of readability, we take the opportunity to survey part of their work along the way.

Acknowledgements. I would like to thank Pierrick Bousseau, Daniel Huybrechts, András Juhász, and Ralph Klaasse for helpful discussions. Special thanks go to Richard Thomas for countless valuable comments and the anonymous referee for many suggestions on improving the exposition. This work was supported by EPSRC grant EP/G06170X/1, "Applied derived categories".

\footnotetext{
${ }^{5}$ The fact that $\beta(\beta-\mathrm{k}) \geq 0$ implies $\beta(\beta-\mathrm{k})=0$ and $q(S)=1$ is a non-trivial result of Dürr-Kabanov-Okonek [6. This fact and its proof are recalled in Section 3 (Proposition 3.1). The number $[\gamma] \in \mathbb{Z}$ for any $\gamma \in H^{2}(S, \mathbb{Z})$ on a surface with $q(S)=1$ is defined as follows. The class $\gamma$ determines an element $\int_{S} \gamma \wedge \cdot \in \Lambda^{2} H^{1}(S, \mathbb{Z})^{*}$. Since $q(S)=1$ we have a canonical isomorphism $\Lambda^{2} H^{1}(S, \mathbb{Z})^{*} \cong \mathbb{Z}$ induced by choosing an integral basis of $H^{1}(S, \mathbb{Z}) \subset H^{1}(S, \mathbb{R})$ compatible with the orientation coming from the complex structure. The integer obtained in this way is denoted by $[\gamma]$.
} 


\section{Poincaré/PT CORRESPONDEnCE}

In this section we give a formula for the virtual cycle $\left[\operatorname{Hilb}^{n}\left(\mathcal{C} / H_{\beta}\right)\right]^{\text {vir }}$ (Proposition 2.1). We then exploit the "product structure" of this formula to prove Main Theorem 1.1, Corollary 1.2, and Theorem 1.3.

2.1. Virtual cycle. Let $\mathcal{C} \subset H_{\beta} \times S \rightarrow H_{\beta}$ be the universal curve over the Hilbert scheme $H_{\beta}=\operatorname{Hilb}_{\beta}(S)$ of effective divisors in class $\beta$. Recall from the introduction that $\operatorname{Hilb}^{n}\left(\mathcal{C} / H_{\beta}\right) \cong P_{\chi}(S, \beta)$ is a component of the $\mathbb{C}^{*}$-fixed locus of the full 3-fold stable pair space $P_{\chi}(X, \beta)$. Also recall that $\chi=1-h+n$, where $h$ is the genus of curves in class $\beta$. We start with the natural embedding

$$
\iota: \operatorname{Hilb}^{n}\left(\mathcal{C} / H_{\beta}\right) \hookrightarrow S^{[n]} \times H_{\beta},
$$

where $S^{[n]}$ is the Hilbert scheme of $n$ points on $S$. A point $(Z, C)$ lies in $\operatorname{Hilb}^{n}\left(\mathcal{C} / H_{\beta}\right)$ if and only if

$$
\left.s_{C}\right|_{Z}=0 \in H^{0}\left(\mathcal{O}_{Z}(C)\right),
$$

where $s_{C}$ is the section cutting out $C \subset S$. The family version of this goes as follows. Let $\mathcal{Z} \subset S^{[n]} \times S$ be the universal subscheme and let

$$
\pi: S^{[n]} \times S \times H_{\beta} \rightarrow S^{[n]} \times H_{\beta}
$$

denote projection. Then

$$
\mathcal{O}(\mathcal{C})^{[n]}:=\pi_{*}\left(\left.\mathcal{O}\left(S^{[n]} \times \mathcal{C}\right)\right|_{\mathcal{Z} \times H_{\beta}}\right)
$$

is a rank $n$ vector bundle on $S^{[n]} \times H_{\beta}$. It has a tautological section $\sigma$ with zero locus $\operatorname{Hilb}^{n}\left(\mathcal{C} / H_{\beta}\right)$. This provides $\operatorname{Hilb}^{n}\left(\mathcal{C} / H_{\beta}\right)$ with a relative perfect obstruction theory over $H_{\beta}$. This construction does not provide an absolute perfect obstruction theory because $H_{\beta}$ can be singular. The notation (5) is chosen for the following reason. Consider projections

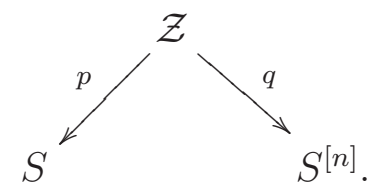

Then for any line bundle $L$ on $S$

$$
L^{[n]}:=q_{*} p^{*} L
$$

is a rank $n$ vector bundle on $S^{[n]}$ known as a tautological bundle (e.g. see [7]). It is not hard to see from the definitions that for any point $p=[C] \in H_{\beta}$

$$
\left.\mathcal{O}(\mathcal{C})^{[n]}\right|_{S^{[n]} \times\{p\}} \cong \mathcal{O}(C)^{[n]}
$$


Dürr-Kabanov-Okonek [6] constructed a natural perfect obstruction theory on $H_{\beta}$ of the form

$$
\left(R \pi_{*} \mathcal{O}_{\mathcal{C}}(\mathcal{C})\right)^{\vee} \rightarrow \mathbb{L}_{H_{\beta}}
$$

In [14, Appendix] this perfect obstruction theory on $H_{\beta}$ and the relative perfect obstruction theory on $\operatorname{Hilb}^{n}\left(\mathcal{C} / H_{\beta}\right)$ are combined to construct an absolute perfect obstruction theory on $\operatorname{Hilb}^{n}\left(\mathcal{C} / H_{\beta}\right)$. See diagram (89) of [14, Appendix] for details. We denote the corresponding virtual cycles on $H_{\beta}$ and $\operatorname{Hilb}^{n}\left(\mathcal{C} / H_{\beta}\right)$ by $\left[H_{\beta}\right]^{\text {vir }}$ and $\left[\operatorname{Hilb}^{n}\left(\mathcal{C} / H_{\beta}\right)\right]^{\text {vir }}$. It is shown in [14, Appendix], that $\left[\operatorname{Hilb}^{n}\left(\mathcal{C} / H_{\beta}\right)\right]^{\text {vir }}$ coincides with the virtual cycle induced by $\mathbb{C}^{*}$-localization of stable pair theory on $X=K_{S}$ to the component $\operatorname{Hilb}^{n}\left(\mathcal{C} / H_{\beta}\right)$ of the $\mathbb{C}^{*}$-fixed locus. Although $H_{\beta}$ can be singular, we still have the following:

Proposition 2.1. For any $S, \beta$

$$
\iota_{*}\left[\operatorname{Hilb}^{n}\left(\mathcal{C} / H_{\beta}\right)\right]^{\text {vir }}=\left(S^{[n]} \times\left[H_{\beta}\right]^{\text {vir }}\right) \cdot c_{n}\left(\mathcal{O}(\mathcal{C})^{[n]}\right)
$$

and its virtual dimension is $v=\frac{\beta(\beta-\mathrm{k})}{2}+n$.

For the proof of this proposition, we need the following lemma.

Lemma 2.2. Let $\pi: M \rightarrow B$ be a flat morphism of $\mathbb{C}$-schemes of finite type with $B$ projective. Let $E^{\bullet} \rightarrow \mathbb{L}_{B}, F^{\bullet} \rightarrow \mathbb{L}_{M}$ be perfect obstruction theories. Suppose that there exists a smooth projective variety $A$ and a rank $r$ vector

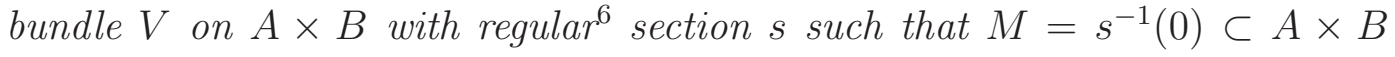
and $\pi: M \rightarrow B$ commutes with projection $\pi_{B}: A \times B \rightarrow B$. This induces a canonical relative perfect obstruction theory $G^{\bullet} \rightarrow \mathbb{L}_{M / B}$ of the form $G^{\bullet}=$ $\left\{\left.\left.V^{*}\right|_{M} \rightarrow \pi_{A}^{*}\left(\Omega_{A}\right)\right|_{M}\right\}$. Suppose there exists an exact triangle

$$
\pi^{*} E^{\bullet} \longrightarrow F^{\bullet} \longrightarrow G^{\bullet}
$$

Denote inclusion by $\iota: M \hookrightarrow A \times B$. Then

$$
\iota_{*}[M]^{\mathrm{vir}}=\left(A \times[B]^{\mathrm{vir}}\right) \cdot c_{r}(V) .
$$

Proof. The content of the lemma is formula (8). For any perfect obstruction theory $F^{\bullet} \rightarrow \mathbb{L}_{M}$ with $M$ projective, the following formula holds [28, Thm. 4.6] (see also [27])

$$
[M]^{\mathrm{vir}}=\left\{s_{\bullet}\left(F^{\bullet \vee}\right) c_{F}(M)\right\}_{v}
$$

Here $s_{\bullet}(\cdot)$ is the total Segre class, $v$ is the virtual dimension of $M$, and $c_{F}(M)$ is Fulton's canonical class which is defined as follows. Take any embedding $M \subset \mathcal{A}$ into a smooth variety $\mathcal{A}$, then

$$
c_{F}(M):=c_{\bullet}\left(\left.T_{\mathcal{A}}\right|_{M}\right) s_{\bullet}\left(C_{M / \mathcal{A}}\right),
$$

\footnotetext{
${ }^{6}$ As defined in [9, B.3.4].
} 
where $C_{M / \mathcal{A}}$ is the normal cone of $M \subset \mathcal{A}$. This definition is independent of choice of embedding [9, Ex. 4.2.6]. Take an embedding $B \subset C$ into a smooth variety and consider

$$
M \subset A \times B \subset A \times C=: \mathcal{A} \text {. }
$$

By (7) we have

$$
s_{\bullet}\left(F^{\bullet \vee}\right)=\pi^{*}\left(s_{\bullet}\left(E^{\bullet \vee}\right)\right) \frac{c_{\bullet}\left(\left.V\right|_{M}\right)}{\left.\pi_{A}^{*}\left(c_{\bullet}\left(T_{A}\right)\right)\right|_{M}} .
$$

Since $M \subset A \times B$ is cut out by a regular section of $V$, we have

$$
\left.C_{M / A \times B} \cong N_{M / A \times B} \cong V\right|_{M} .
$$

Consider the following short exact sequence of cones

$$
\left.N_{M / A \times B} \longrightarrow C_{M / A \times C} \longrightarrow C_{A \times B / A \times C}\right|_{M} .
$$

We deduce

$$
c_{F}(M)=\left.\pi_{A}^{*}\left(c_{\bullet}\left(T_{A}\right)\right)\right|_{M} \pi^{*}\left(c_{\bullet}\left(\left.T_{C}\right|_{B}\right)\right) \frac{\pi^{*} s_{\bullet}\left(C_{B / C}\right)}{c_{\bullet}\left(\left.V\right|_{M}\right)} .
$$

Formula (9) therefore implies

$$
[M]^{\mathrm{vir}}=\left\{\pi^{*}\left(s_{\bullet}\left(E^{\bullet \vee}\right) c_{\bullet}\left(\left.T_{C}\right|_{B}\right) s_{\bullet}\left(C_{B / C}\right)\right)\right\}_{v}=\pi^{*}[B]^{\mathrm{vir}}
$$

where the second equality follows from applying (9) to $E^{\bullet} \rightarrow \mathbb{L}_{B}$. The projection formula gives

$$
\iota_{*}[M]^{\mathrm{vir}}=\left(A \times[B]^{\mathrm{vir}}\right) \cdot \iota_{*}[M] .
$$

Since $M \subset A \times B$ is cut out by a regular section of $V$, we have $\iota_{*}[M]=c_{r}(V)$ [9, Prop. 14.1] and the proposition is proved.

Proof of Proposition [2.1. Diagram (89) of [14, Appendix] provides the required exact triangle. It is left to show $\operatorname{Hilb}^{n}\left(\mathcal{C} / H_{\beta}\right) \rightarrow H_{\beta}$ is flat and the tautological section $\sigma$ of $\mathcal{O}(\mathcal{C})^{[n]}$ is regular. The fibre of the morphism $\operatorname{Hilb}^{n}\left(\mathcal{C} / H_{\beta}\right) \rightarrow H_{\beta}$ over $C \in H_{\beta}$ is $C^{[n]}$, i.e. the Hilbert scheme of $n$ points on the effective divisor $C$. The scheme $C^{[n]}$ is cut out by a tautological section of $L^{[n]}$ where $L:=\mathcal{O}(C)$. Moreover, $C^{[n]} \subset S^{[n]}$ has codimension $n$ (see [14, Footnote 18], which uses [1, 13]). Therefore $\left.\sigma\right|_{S^{[n]} \times\{C\}}$ is regular for all $C \in H_{\beta}$. From this one can deduce that $\operatorname{Hilb}^{n}\left(\mathcal{C} / H_{\beta}\right) \rightarrow H_{\beta}$ is flat and $\sigma$ is regular. 
2.2. Relation to Poincaré invariants. In Section 1 we introduced the stable pair invariants (11)

$$
P_{\chi, \beta}\left(X, \tau_{\alpha_{1}}\left(\sigma_{1}\right) \cdots \tau_{\alpha_{m}}\left(\sigma_{m}\right)\right)
$$

and the contribution to these invariants of the component $P_{\chi}(S, \beta) \cong \operatorname{Hilb}^{n}\left(\mathcal{C} / H_{\beta}\right)$ of the $\mathbb{C}^{*}$-fixed locus

$$
P_{\chi, \beta}\left(S, \tau_{\alpha_{1}}\left(\sigma_{1}\right) \cdots \tau_{\alpha_{m}}\left(\sigma_{m}\right)\right)
$$

We only consider the case of primary point insertions

$$
P_{\chi, \beta}\left(S, \tau_{0}(\mathrm{pt})^{m}\right)=\int_{\left[P_{\chi}(S, \beta)\right] \operatorname{vir}} \frac{1}{e\left(N^{\text {vir }}\right)} \tau_{0}(\mathrm{pt})^{m} .
$$

In the case $n=0, \operatorname{Hilb}^{n}\left(\mathcal{C} / H_{\beta}\right) \cong H_{\beta}$ and $\left[H_{\beta}\right]^{\text {vir }}$ was introduced many years ago by Dürr-Kabanov-Okonek [6, Def. 3.1]. They used this virtual cycle to define Poincaré invariants. We recall their definition. Consider the two Abel-Jacobi maps

$$
\begin{aligned}
& \mathrm{AJ}^{+}: H_{\beta} \rightarrow \operatorname{Pic}^{\beta}(S), \\
& \mathrm{AJ}^{-}: H_{\mathrm{k}-\beta} \rightarrow \operatorname{Pic}^{\mathrm{k}-\beta}(S) \cong \operatorname{Pic}^{\beta}(S),
\end{aligned}
$$

where $\operatorname{Pic}^{\mathrm{k}-\beta}(S) \cong \operatorname{Pic}^{\beta}(S), L \mapsto L^{*} \otimes K_{S}$. Then the Poincaré invariants are

$$
\begin{gathered}
P_{S}^{+}(\beta):=\mathrm{AJ}_{*}^{+}\left(\sum_{i} c_{1}\left(\left.\mathcal{O}(\mathcal{C})\right|_{H_{\beta} \times\{\mathrm{pt}\}}\right)^{i} \cap\left[H_{\beta}\right]^{\mathrm{vir}}\right), \\
P_{S}^{-}(\beta):=(-1)^{\chi\left(\mathcal{O}_{S}\right)+\frac{\beta(\beta-\mathrm{k})}{2}} \mathrm{AJ}_{*}^{-}\left(\sum_{i}(-1)^{i} c_{1}\left(\left.\mathcal{O}(\mathcal{C})\right|_{H_{\mathrm{k}-\beta} \times\{\mathrm{pt}\}}\right)^{i} \cap\left[H_{\mathrm{k}-\beta}\right]^{\mathrm{vir}}\right) .
\end{gathered}
$$

In the first line, $\mathcal{C}$ denotes the universal curve over $H_{\beta}$ and in the second line, the universal curve over $H_{\mathrm{k}-\beta}$. Note that $P_{S}^{ \pm}(\beta) \in \Lambda^{*} H^{1}(S, \mathbb{Z})^{*}$. We write the (numerical) degree $2 q(S)$ part of $P_{S}^{+}(\beta) \in \Lambda^{*} H^{1}(S, \mathbb{Z})^{*}$ by

$$
P_{S}(\beta) \in \mathbb{Z} \text {. }
$$

The product structure of the virtual cycle of Proposition 2.1 leads to Main Theorem 1.1 of the introduction:

Proof of Theorem 1.1. We want to calculate the invariant

$$
P_{\chi, \beta}\left(S, \tau_{0}(\mathrm{pt})^{m}\right):=\frac{1}{e\left(N^{\text {vir }}\right)} \tau_{0}(\mathrm{pt})^{m} \cap\left[\operatorname{Hilb}^{n}\left(\mathcal{C} / H_{\beta}\right)\right]^{\mathrm{vir}},
$$

\footnotetext{
${ }^{7}$ From the construction the Poincaré invariants take values in homology $H_{*}\left(\operatorname{Pic}^{\beta}(S)\right) \cong$ $\Lambda^{*} H^{1}(S, \mathbb{Z})$. We use Poincaré duality so the invariants take values in cohomology $\Lambda^{*} H^{1}(S, \mathbb{Z})^{*}$.
} 
where $\operatorname{Hilb}^{n}\left(\mathcal{C} / H_{\beta}\right) \cong P_{\chi}(S, \beta)$, and $\chi$ and $n$ are related by $\chi=1-h+n$ (Section 1). Let $\varpi: \operatorname{Hilb}^{n}\left(\mathcal{C} / H_{\beta}\right) \rightarrow H_{\beta}$ denote projection, then we claim

$$
\tau_{0}(\mathrm{pt})=\varpi^{*} c_{1}\left(\left.\mathcal{O}(\mathcal{C})\right|_{H_{\beta} \times\{\mathrm{pt}\}}\right) .
$$

The proof can be found in [15, Proof Cor. 4.2], but we quickly reproduce it here. Consider the Cartesian diagram

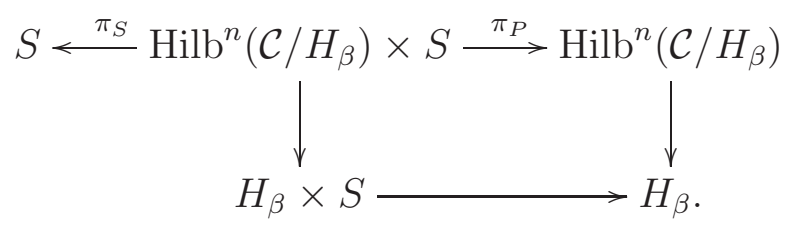

By the definition (2),$\tau_{0}(\mathrm{pt})=\pi_{P *}\left(\pi_{S}^{*}[\mathrm{pt}] \cdot c_{1}(\mathbb{F})\right)$, where $\mathbb{F}$ is the universal sheaf on $\operatorname{Hilb}^{n}\left(\mathcal{C} / H_{\beta}\right) \times S$. Hence (12) follows from the fact that $c_{1}(\mathbb{F})$ is the pull-back of $c_{1}(\mathcal{O}(\mathcal{C}))$ from $H_{\beta} \times S$ and going around the Cartesian diagram.

In order to calculate $e\left(N^{\text {vir }}\right)$, we use a formula for the $\mathbb{C}^{*}$-equivariant $K$ theory class of $N^{\mathrm{vir}}$ from [15]. Consider the projections

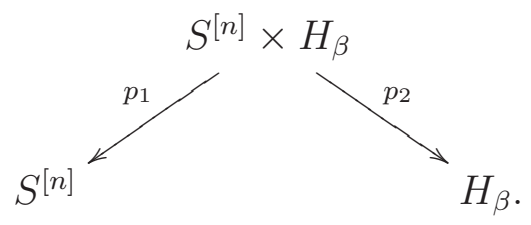

Then [15, Eqn. (12)] reads

$$
\left[N^{\mathrm{vir}}\right]=\left.\left[\left(\mathcal{O}(\mathcal{C})^{[n]}\right)^{*}-p_{1}^{*} \Omega_{S^{[n]}}-p_{2}^{*}\left(R \pi_{*} \mathcal{O}_{\mathcal{C}}(\mathcal{C})\right)^{\vee}\right]\right|_{\operatorname{Hilb}^{n}\left(\mathcal{C} / H_{\beta}\right)} \otimes \mathfrak{t}
$$

where $\mathfrak{t}$ is the irreducible representation of $\mathbb{C}^{*}$ of weight 1 . Recall from (5) that $\mathcal{O}(\mathcal{C})^{[n]}$ is a vector bundle on $S^{[n]} \times H_{\beta}, R \pi_{*} \mathcal{O}_{\mathcal{C}}(\mathcal{C})$ is a complex on $H_{\beta}$, and $\pi$ denotes projection $H_{\beta} \times S \rightarrow H_{\beta}$. By pushing forward along the inclusion $\iota: \operatorname{Hilb}^{n}\left(\mathcal{C} / H_{\beta}\right) \hookrightarrow S^{[n]} \times H_{\beta}$ and using (11), (12), (13), we see that $P_{\chi, \beta}\left(S, \tau_{0}(\mathrm{pt})^{m}\right)$ equals

$\frac{e\left(p_{1}^{*} \Omega_{S^{[n]}} \otimes \mathfrak{t}\right) \cdot e\left(p_{2}^{*}\left(R \pi_{*} \mathcal{O}_{\mathcal{C}}(\mathcal{C})\right)^{\vee} \otimes \mathfrak{t}\right)}{e\left(\left(\mathcal{O}(\mathcal{C})^{[n]}\right)^{*} \otimes \mathfrak{t}\right)} \cdot \varpi^{*} c_{1}\left(\left.\mathcal{O}(\mathcal{C})\right|_{H_{\beta} \times\{\mathrm{pt}\}}\right)^{m} \cap \iota_{*}\left[\operatorname{Hilb}^{n}\left(\mathcal{C} / H_{\beta}\right)\right]^{\text {vir }}$.

Next we want to use the formula for $\iota_{*}\left[\operatorname{Hilb}^{n}\left(\mathcal{C} / H_{\beta}\right)\right]^{\text {vir }}$ from Proposition 2.1 . Recall from the assumptions of the theorem that $m:=\frac{\beta(\beta-\mathrm{k})}{2}$. Since the virtual dimension of $\left[H_{\beta}\right]^{\text {vir }}$ is also $\frac{\beta(\beta-\mathrm{k})}{2}$, the cycle

$$
c_{1}\left(\left.\mathcal{O}(\mathcal{C})\right|_{H_{\beta} \times\{\mathrm{pt}\}}\right)^{m} \cap\left[H_{\beta}\right]^{\text {vir }}
$$


is 0 -dimensional and can be written as $\sum_{i} \mu_{i} p_{i}$, where $\mu_{i}$ are integers and $p_{i}=\left[C_{i}\right] \in H_{\beta}$ are points. Then

$$
P_{S}(\beta)=\sum_{i} \mu_{i}
$$

by definition of the Poincaré invariants (10). Therefore $P_{\chi, \beta}\left(S, \tau_{0}(\mathrm{pt})^{m}\right)$ equals

$$
\left.\sum_{i} \mu_{i} \int_{S^{[n]}} \frac{e\left(p_{1}^{*} \Omega_{S^{[n]}} \otimes \mathfrak{t}\right) \cdot e\left(p_{2}^{*}\left(R \pi_{*} \mathcal{O}_{\mathcal{C}}(\mathcal{C})\right)^{\vee} \otimes \mathfrak{t}\right)}{e\left(\left(\mathcal{O}(\mathcal{C})^{[n]}\right)^{*} \otimes \mathfrak{t}\right)} c_{n}\left(\mathcal{O}(\mathcal{C})^{[n]}\right)\right|_{S^{[n]} \times\left\{p_{i}\right\}} .
$$

In order to go from equivariant Euler classes to Chern classes we use the following formula (e.g. [15, Eqn. (16)]). For any complex $E$ of rank $r$

$$
e(E \otimes \mathfrak{t})=t^{r} c_{-1 / t}\left(E^{\vee}\right)
$$

where $c_{x}(E)=1+c_{1}(E) x+c_{2}(E) x^{2}+\cdots$ is the total Chern class and $t:=c_{1}(\mathfrak{t})$ is the equivariant parameter. Define $L_{i}:=\mathcal{O}_{S}\left(C_{i}\right)$, where $p_{i}=\left[C_{i}\right] \in H_{\beta}$ was introduced earlier in the proof. Then (6) implies

$$
\left.\mathcal{O}(\mathcal{C})^{[n]}\right|_{S^{[n]} \times\left\{p_{i}\right\}} \cong L_{i}^{[n]}
$$

Similarly

$$
\left.p_{2}^{*} R \pi_{*} \mathcal{O}_{\mathcal{C}}(\mathcal{C})\right|_{S^{[n]} \times\left\{p_{i}\right\}} \cong R \Gamma\left(\mathcal{O}_{C_{i}}\left(C_{i}\right)\right) \otimes \mathcal{O}
$$

Using (14), (15), (16) shows that $P_{\chi, \beta}\left(S, \tau_{0}(\mathrm{pt})^{m}\right)$ equals

$$
\begin{aligned}
& \sum_{i} \mu_{i} \int_{S^{[n]}} \frac{t^{2 n} c_{-1 / t}\left(T_{S^{[n]}}\right) \cdot t^{1-h+\beta^{2}} c_{-1 / t}\left(R \Gamma\left(\mathcal{O}_{C_{i}}\left(C_{i}\right)\right) \otimes \mathcal{O}\right)}{t^{n} c_{-1 / t}\left(L_{i}^{[n]}\right)} c_{n}\left(L_{i}^{[n]}\right) \\
& =\sum_{i} \mu_{i} \int_{S^{[n]}} t^{n+m}\left(\frac{-1}{t}\right)^{n} \frac{c_{\bullet}\left(T_{S^{[n]}}\right)}{c_{\bullet}\left(L_{i}^{[n]}\right)} c_{n}\left(L_{i}^{[n]}\right) \\
& =(-1)^{n} t^{m} \sum_{i} \mu_{i} \int_{S^{[n]}} \frac{c_{\bullet}\left(T_{S^{[n]}}\right)}{c_{\bullet}\left(L_{i}^{[n]}\right)} c_{n}\left(L_{i}^{[n]}\right),
\end{aligned}
$$

where the second equality uses $m:=\frac{\beta(\beta-\mathrm{k})}{2}$ and the factor $(-1 / t)^{n}$ arises from the fact that $c_{n}\left(L_{i}^{[n]}\right)$ has degree $n$ and $S^{[n]}$ has dimension $2 n$.

By [7], for each $n$ there exists a universal polynomial $P_{n}\left(x_{1}, x_{2}, x_{3}, x_{4}\right)$ such that for all $i$ we have

$$
P_{n}\left(c_{1}\left(L_{i}\right)^{2}, c_{1}\left(L_{i}\right) \cdot \mathbf{k}, \mathrm{k}^{2}, c_{2}(S)\right)=\int_{S^{[n]}} c_{n}\left(L_{i}^{[n]}\right) \frac{c_{\bullet}\left(T_{S^{[n]}}\right)}{c_{\bullet}\left(L_{i}^{[n]}\right)} .
$$


Since $c_{1}\left(L_{i}\right)=\beta$ for all $i$, all these integrals are the same. Using $P_{S}(\beta)=\sum_{i} \mu_{i}$, formula (17) becomes

$$
P_{\chi, \beta}\left(S, \tau_{0}(\mathrm{pt})^{m}\right)=(-1)^{n} t^{m} P_{S}(\beta) \int_{S^{[n]}} c_{n}\left(L^{[n]}\right) \frac{c_{\bullet}\left(T_{S^{[n]}}\right)}{c_{\bullet}\left(L^{[n]}\right)},
$$

where $L:=L_{i}$ for arbitrary choice of $i$.

For any $S, L$, the integral in (18) is given by $P_{n}\left(c_{1}(L)^{2}, c_{1}(L) \cdot \mathrm{k}, \mathrm{k}^{2}, c_{2}(S)\right)$. For any $S, L$ with the additional property that $L$ is globally generated, we can compute the integral in (18). If $L$ is globally generated, we can write $L=\mathcal{O}(C)$ for a smooth curve $C \subset S$. Then the Hilbert scheme $C^{[n]}$ of $n$ points on $C$ is cut out smoothly and transversally by a tautological section of $L^{[n]}$. Hence

$$
\int_{S^{[n]}} c_{n}\left(L^{[n]}\right) \frac{c_{\bullet}\left(T_{S^{[n]}}\right)}{c_{\bullet}\left(L^{[n]}\right)}=\int_{C^{[n]}} c_{n}\left(T_{C^{[n]}}\right)=e\left(C^{[n]}\right) .
$$

These Euler characteristics are given by the well-known expression

$$
\sum_{n=0}^{\infty} e\left(C^{[n]}\right) q^{n}=(1-q)^{2 g-2}
$$

where $g$ is the genus of $C$. We conclude that

$$
P_{n}\left(c_{1}(L)^{2}, c_{1}(L) \cdot \mathrm{k}, \mathrm{k}^{2}, c_{2}(S)\right)=\int_{S^{[n]}} c_{n}\left(L^{[n]}\right) \frac{c_{\bullet}\left(T_{S^{[n]}}\right)}{c_{\bullet}\left(L^{[n]}\right)}=(-1)^{n}\left(\begin{array}{c}
2 g-2 \\
n
\end{array}\right),
$$

where $2 g-2=c_{1}(L)^{2}+c_{1}(L) \cdot \mathbf{k}$.

Since (19) holds for any $S, L$ with $L$ globally generated and $P_{n}$ is polynomial, it holds for any $S, L$. The theorem follows by combining (18) and (19).

2.3. Application to Seiberg-Witten invariants. Dürr-Kabanov-Okonek conjectured that Poincaré invariants (10) are equal to Seiberg-Witten invariants from 4-manifold theory [6, Conj. 5.3]. Using a wall-crossing formula and blow-up formula for $P_{S}^{ \pm}(\beta)$, they reduced their conjecture to a purely algebraic statement about $H_{\mathrm{k}}$, which was proved by Chang-Kiem [4]. By these (non-trivial!) results, we can write the degree $2 q(S)$ part of $P_{S}^{+}(\beta)$ as

$$
P_{S}(\beta)=S W(\beta) \in \mathbb{Z},
$$

where $S W(\beta)$ are the original Seiberg-Witten invariant of $S, \beta$ (see [32, 20]). Combining the Poincaré/PT correspondence of Theorem 1.1 with the (much deeper!) Poincaré/SW correspondence of [6, 4, gives Corollary 1.2. An application of this corollary is that for $S, \beta$ with $\beta$ irreducible and $m=\frac{\beta(\beta-\mathrm{k})}{2}$ point insertions the GW/PT correspondence encodes (a very special case of) Taubes' GW/SW correspondence [29, 30]. This is the content of Theorem 1.3 of the introduction: 
Proof of Theorem 1.3. Since $\beta$ is irreducible, $P_{\chi}(X, \beta)^{\mathbb{C}^{*}} \cong P_{\chi}(S, \beta)$ for all $\chi$. Hence $P_{\beta}\left(X, \tau_{0}(\mathrm{pt})^{m}\right)=P_{\beta}\left(S, \tau_{0}(\mathrm{pt})^{m}\right)$ and the result follows from Theorem 1.1. Note that the equivariant parameter $t$ of the leading term of both generating functions match by [14, Lem. 3.3].

Remark 2.3. The following is a variation on Theorem 1.3. Fix any $S, \beta$ such that $-K_{S}$ is nef and $\beta$ is sufficiently ample. Assume the GW/PT correspondence ${ }^{9}$ holds for $\mathbf{Z}_{\beta}^{G W}\left(X, \tau_{0}(\mathrm{pt})^{m}\right), \mathbf{Z}_{\beta}^{P}\left(X, \tau_{0}(\mathrm{pt})^{m}\right)$. Also assume that the BPS spectrum of $X$ is finite 10 . Then

$$
\begin{aligned}
& \mathrm{Z}_{\beta}^{G W}\left(X, \tau_{0}(\mathrm{pt})^{m}\right)=t^{m} S W(\beta)(2 \sin (u / 2))^{2 h-2}, \\
& S W(\beta)=\int_{\left[\bar{M}_{h, m}^{\prime}(S, \beta)\right]^{\mathrm{vir}}} \prod_{i=1}^{m} \mathrm{ev}_{i}^{*}[\mathrm{pt}] .
\end{aligned}
$$

The proof goes as follows. Since $h \geq 1$ and the BPS spectrum is assumed finite, applying the coordinate transformation $-q=e^{i u}$ to $Z_{\beta}^{G W}\left(X, \tau_{0}(\mathrm{pt})^{m}\right)$ gives a Laurent polynomial in $q$. Moreover, it is symmetric under $q \leftrightarrow q^{-1}$, so of the form

$$
a_{b} q^{-b}+a_{b-1} q^{-(b-1)}+\cdots+a_{b-1} q^{b-1}+a_{b} q^{b},
$$

for some $b \geq 0$. By [14, Prop. 5.1], we have $P_{\chi}(X, \beta)^{\mathbb{C}^{*}} \cong P_{\chi}(S, \beta)$ for all $\chi \leq h-1$. Combining this with Theorem 1.1 and (20) gives the result.

Remark 2.4. One can speculate that for any algebraic $S, \beta$, Taubes' GW/SW correspondence follows from the GW/PT correspondence. This requires dealing with other components of $P_{\chi}(X, \beta)^{\mathbb{C}^{*}}$. Conversely, one can try to derive cases of the GW/PT correspondence for $X=K_{S}$ from Taubes' GW/SW correspondence as is done in Theorem 1.3. These are interesting questions for future research.

\section{WALL-CROSSING AND DUALITY}

In this section we study the stable pair invariants $P_{\chi, \beta}\left(S, \tau_{0}(\mathrm{pt})^{m}\right)$ for any $m$ and any surface $S$ with $p_{g}(S)=0$. The results of [15] (recalled in Theorem A.2 of Appendix A suggest that these invariants are always given by universal functions in the topological numbers $\beta^{2}, \beta . c_{1}(S), c_{1}(S)^{2}, c_{2}(S)$ and certain invariants of the ring structure of $H^{1}(S, \mathbb{Z})$. In Appendix $\mathbb{B}$ we show that this is not the case. The reason is that $P_{\chi, \beta}\left(S, \tau_{0}(\mathrm{pt})^{m}\right)$ is related to a Poincaré

\footnotetext{
${ }^{8}$ I.e. $\beta$ such that $h \geq 1$ and $\beta$ is $(4 h-3)$-very ample [15. Prop. 5.1].

${ }^{9}$ The GW/PT correspondence has been proved in many cases [18, 19, 21, 22.

${ }^{10}$ I.e. after writing $Z_{\beta}^{G W}\left(X, \tau_{0}(\mathrm{pt})^{m}\right)$ in BPS form [10, 11], 23, Eqn. (3.13)], we assume there are only finitely many nonzero $n_{g, \beta^{\prime}}$.
} 
invariant by Main Theorem 1.1 and it is easy to cook up surfaces $S$ with $p_{g}(S)=$ 0 whose Poincaré invariants are not given by universal functions (Examples B.1. B.3, B.5 of Appendix B).

However, Dürr-Kabanov-Okonek prove that when $p_{g}(S)=0$ the difference of Poincaré invariants in class $\beta$ and $\mathrm{k}-\beta$ satisfies a universal formula. Combining their formula with Main Theorem 1.1 gives an expression for the difference of $P_{\chi, \beta}\left(S, \tau_{0}(\mathrm{pt})^{m}\right)$ and $P_{\chi, \mathrm{k}-\beta}\left(S, \tau_{0}(\mathrm{pt})^{m}\right)$. This is Theorem 1.4 of the introduction and the second application of Main Theorem 1.1.

3.1. Dürr-Kabanov-Okonek's wall-crossing. We recall the wall-crossing formula for Poincaré invariants [6, Thm. 3.16]. Since we use this formula to establish Theorem 1.4, and for the sake of completeness, we recall DürrKabanov-Okonek's interesting argument. Moreover, their results lead to a nice observation about the reduced virtual cycle for stable pairs, which is of independent interest (Proposition A.1 in Appendix A). The results and arguments presented in this section come entirely from their paper [6].

The following is contained in [6, Lem. 2.17] and its proof (see also [6, Cor. 3.15]).

Proposition 3.1 (Dürr-Kabanov-Okonek). Let $S$ be any surface. Suppose that $\beta$ satisfies the following conditions:

(i) For any effective $L \in \operatorname{Pic}^{\beta}(S)$ with $c_{1}(L)=\beta$ we have $H^{2}(L)=0$. Note: this is automatic when $p_{g}(S)=0$.

(ii) $\beta(\beta-\mathrm{k}) \geq 0$.

(iii) $H_{\beta}$ and $H_{\mathrm{k}-\beta}$ are both non-empty.

Then $\beta(\beta-\mathbf{k})=0$ and $\chi\left(\mathcal{O}_{S}\right)=0$. Note: $\chi\left(\mathcal{O}_{S}\right)=0$ is equivalent to $q(S)=1$ when $p_{g}(S)=0$.

Proof. The result follows by showing

$$
\frac{\beta(\beta-\mathbf{k})}{2}+\chi\left(\mathcal{O}_{S}\right)=0 \text { and } \chi\left(\mathcal{O}_{S}\right) \geq 0 \text {. }
$$

Let $p: \operatorname{Pic}^{\beta}(S) \times S \rightarrow \operatorname{Pic}^{\beta}(S)$ denote projection and let $\mathscr{P}$ be a choice of Poincaré bundle on $\operatorname{Pic}^{\beta}(S) \times S$.

Condition (i) is equivalent to the statement that the images (Brill-Noether loci) of the two maps $H_{\mathrm{k}-\beta} \rightarrow \operatorname{Pic}^{\mathrm{k}-\beta}(S) \cong \operatorname{Pic}^{\beta}(S)$ and $H_{\beta} \rightarrow \operatorname{Pic}^{\beta}(S)$ are disjoint. In other words, their complements $U$ and $V$ satisfy $\operatorname{Pic}^{\beta}(S)=U \cup V$. Moreover, for any $L \in \operatorname{Pic}^{\beta}(S)$, we have $H^{2}(L)=0$ when $L \in U$ and $H^{0}(L)=0$ when $L \in V$. In other words

$$
\left.R^{2} p_{*} \mathscr{P}\right|_{U}=0,\left.R^{0} p_{*} \mathscr{P}\right|_{V}=0 .
$$


This implies

$$
\operatorname{rk} R p_{*} \mathscr{P}=\frac{\beta(\beta-\mathrm{k})}{2}+\chi\left(\mathcal{O}_{S}\right) \leq 0 .
$$

Since $H_{\beta}$ and $H_{\mathrm{k}-\beta}$ are both non-empty (Condition (iii)), $S$ cannot be rational because otherwise we get a section of $K_{S}$. Moreover, $S$ cannot be ruled: for $F$ the class of a fibre either $\beta$. $[F]<0$ in which case $H_{\beta}=\varnothing$ or $\beta$. $[F] \geq 0$ in which case $(\mathrm{k}-\beta) \cdot[F]<0$ so $H_{\mathrm{k}-\beta}=\varnothing$. Similarly, $S$ cannot be the blowup of a ruled surface. We conclude that the Kodaira dimension of $S$ is $\geq 0$. Therefore $\chi\left(\mathcal{O}_{S}\right) \geq 0$ and

$$
\frac{\beta(\beta-\mathbf{k})}{2}+\chi\left(\mathcal{O}_{S}\right) \geq 0
$$

Theorem 3.2 (Dürr-Kabanov-Okonek). Let $S$ be a surface with $p_{g}(S)=0$. Let $\mathscr{P}$ be a choice of normalized Poincaré bundle on $\operatorname{Pic}^{\beta}(S)$, i.e. $\left.\mathscr{P}\right|_{\operatorname{Pic}^{\beta}(S) \times\{\mathrm{pt}\}} \cong$ $\mathcal{O}$. Denote projection by $p: \operatorname{Pic}^{\beta}(S) \times S \rightarrow \operatorname{Pic}^{\beta}(S)$. Then

$$
P_{S}^{+}(\beta)-P_{S}^{-}(\beta)=\sum_{i \geq 1-\chi(\beta)} s_{i}\left(p_{!} \mathscr{P}\right),
$$

where $1-\chi(\beta)=q(S)-\frac{\beta(\beta-\mathrm{k})}{2}$.

Proof. We first note that $\beta$ satisfies Condition (44) of the introduction if and only if $H_{\mathrm{k}-\beta}=\varnothing$. Indeed if $\beta$ satisfies Condition (41) we clearly have $H_{\mathrm{k}-\beta}=0$. Conversely $H_{\mathrm{k}-\beta}=\mathbb{P}\left(R^{2} p_{*} \mathscr{P}\right)$ by [6, Lem. 2.15], so if $H_{\mathrm{k}-\beta}=\varnothing$ we have $R^{2} p_{*} \mathscr{P}=0$ and hence $\beta$ satisfies Condition (44) by cohomology and base change. Similarly $\mathrm{k}-\beta$ satisfies Condition (4) if and only if $H_{\beta}=\varnothing$ (using $H_{\beta}=\mathbb{P}\left(R^{2} p_{*} \mathscr{P}^{*}\left(K_{S}\right)\right)$ [6, Lem. 2.15]).

The rest of the proof of [6] runs as follows. If $\frac{\beta(\beta-\mathrm{k})}{2}<0$, then the virtual dimension of $H_{\beta}$ and $H_{\mathrm{k}-\beta}$ are negative so the LHS is zero. Moreover the RHS is zero because of degree reasons $\left(\operatorname{Pic}^{\beta}(S)\right.$ has dimension $\left.q(S)\right)$. For the remainder of the proof assume $\frac{\beta(\beta-\mathrm{k})}{2} \geq 0$.

Let $\mathscr{P}$ be a choice of Poincaré bundle on $\operatorname{Pic}^{\beta}(S) \times S$ and let

$$
p: \operatorname{Pic}^{\beta}(S) \times S \rightarrow \operatorname{Pic}^{\beta}(S)
$$

denote projection. In Appendix $\mathrm{A}$ we describe a construction, which embeds $H_{\beta}$ into a smooth ambient space in a natural way. For sufficiently ample divisor $A \subset S$ define $\gamma:=[A]+\beta$ and let $\mathcal{Q}$ be a choice of Poincaré bundle on $\operatorname{Pic}^{\gamma}(S) \times S$. Again we denote projection by $p: \operatorname{Pic}^{\gamma}(S) \times S \rightarrow \operatorname{Pic}^{\gamma}(S)$. By sufficient ampleness of $A$, the Abel-Jacobi map

$$
\text { AJ }: H_{\gamma} \longrightarrow \operatorname{Pic}^{\gamma}(S)
$$


is a projective bundle and $H_{\gamma} \cong \mathbb{P}\left(p_{*} \mathcal{Q}\right)$. Moreover we can embed $H_{\beta} \hookrightarrow H_{\gamma}$ by adding the divisor $A$. There exists a natural sheaf $F$ on $H_{\gamma}$ with tautological section cutting out $H_{\beta} \hookrightarrow H_{\gamma}$. Since $p_{g}(S)=0$, the sheaf $F$ is a vector bundle on a Zariski open neighbourhood of $H_{\beta}$. See Appendix $\mathrm{A}$ for the details. Let $r$ be the rank of $F$ and let $h:=c_{1}(\mathcal{O}(1))$ on $\mathbb{P}\left(p_{*} \mathcal{Q}\right)$. If $H_{\beta} \neq \varnothing$, then

$$
\iota_{*}\left[H_{\beta}\right]^{\mathrm{vir}}=c_{r}(F)
$$

on $H_{\gamma}$ (Proposition A.1 of Appendix $\mathrm{A}$ for $n=0$ ) and

$$
\mathrm{AJ}_{*}\left(c_{1}\left(\left.\mathcal{O}(\mathcal{C})\right|_{H_{\beta} \times\{\mathrm{pt}\}}\right)^{i} \cap\left[H_{\beta}\right]^{\mathrm{vir}}\right)=\mathrm{AJ}_{*}\left(c_{r}(F) h^{i}\right) .
$$

A similar formula holds for $\left[H_{\mathrm{k}-\beta}\right]^{\mathrm{vir}}$ when $H_{\mathrm{k}-\beta} \neq \varnothing$. Moreover by [6, Prop. 2.18] (or [15, Lem. 4.3])

$$
\mathrm{AJ}_{*}\left(c_{r}(F) h^{i}\right)=s_{i-\chi(\beta)+1}\left(\tau_{\leq 1} p_{!} \mathscr{P}\right),
$$

where $\chi(\beta)$ denotes the holomorphic Euler characteristic of $\beta$. Equation (22) also holds when $H_{\beta}=\varnothing$.

If $\beta$ satisfies Condition (41) (i.e. $H_{\mathrm{k}-\beta}=\varnothing$ ), then $F$ is a vector bundle on $H_{\gamma}$, and $s_{i}\left(p_{!} \mathscr{P}\right)=s_{i}\left(\tau_{\leq 1} p_{!} \mathscr{P}\right)$. The formula follows from (22) and (21). If $\mathrm{k}-\beta$ satisfies Condition (4) (i.e. $H_{\beta}=\varnothing$ ), then the formula follows similarly using Serre duality $R p_{*} \mathscr{P}^{*}\left(K_{S}\right) \cong\left(R p_{*} \mathscr{P}[2]\right)^{\vee}$.

We are left with the case where neither $\beta$ nor $\mathrm{k}-\beta$ satisfies Condition (4), i.e. $H_{\beta}$ and $H_{\mathrm{k}-\beta}$ are both non-empty. The wall-crossing formula is equivalent to

$$
\sum_{i \geq 1-\chi(\beta)}\left(s_{i}\left(\tau_{\leq 1} p_{!} \mathscr{P}\right)+(-1)^{i} s_{i}\left(\tau_{\leq 1} p_{!} \mathscr{P}^{*}\left(K_{S}\right)\right)\right)=\sum_{i \geq 1-\chi(\beta)} s_{i}\left(p_{!} \mathscr{P}\right) .
$$

By Proposition 3.1, $\beta(\beta-\mathrm{k}) \geq 0$ and $H_{\beta}, H_{\mathrm{k}-\beta}$ are both non-empty implies $\chi\left(\mathcal{O}_{S}\right)=0$ and $\beta(\beta-\mathrm{k})=0$. Since $p_{g}(S)=0$, we have $q(S)=1$. Since $s_{1}\left(\tau_{\leq 1} p_{!} \mathscr{P}\right)=c_{1}\left(R^{1} p_{*} \mathscr{P}\right)-c_{1}\left(p_{*} \mathscr{P}\right)$, it suffices to show

$$
s_{1}\left(\tau_{\leq 1} p_{!} \mathscr{P}^{*}\left(K_{S}\right)\right)=c_{1}\left(R^{2} p_{*} \mathscr{P}\right) .
$$

Take a locally free resolution $\left[E^{0} \stackrel{d^{0}}{\rightarrow} E^{1} \stackrel{d^{1}}{\rightarrow} E^{2}\right]$ of $R p_{*} \mathscr{P}$. Then Serre duality $R p_{*} \mathscr{P}^{*}\left(K_{S}\right) \cong\left(R p_{*} \mathscr{P}[2]\right)^{\vee}$ implies

$$
\begin{aligned}
s_{1}\left(\tau_{\leq 1} p_{!} \mathscr{P}^{*}\left(K_{S}\right)\right) & =c_{1}\left(\operatorname{ker}\left(d^{0 *}\right)\right)-c_{1}\left(E^{2 *}\right)=c_{1}\left(E^{2}\right)+c_{1}\left(\left(\operatorname{coker} d^{0}\right)^{*}\right), \\
c_{1}\left(R^{2} p_{*} \mathscr{P}\right) & =c_{1}\left(E^{2}\right)-c_{1}\left(\operatorname{im} d^{1}\right)=c_{1}\left(E^{2}\right)+c_{1}\left(\left(E^{1} / \operatorname{ker} d^{1}\right)^{*}\right) .
\end{aligned}
$$

In the proof of Proposition 3.1 we saw that $R^{1} p_{*} \mathscr{P}$ is torsion. Dualizing the short exact sequence

$$
0 \rightarrow R^{1} p_{*} \mathscr{P} \rightarrow \text { coker } d^{0} \rightarrow E^{1} / \text { ker } d^{1} \rightarrow 0
$$

shows $\left(\operatorname{coker} d^{0}\right)^{*} \cong\left(E^{1} / \operatorname{ker} d^{1}\right)^{*}$. This proves the desired result. 
Proof of Theorem 1.4. Fix $S, \beta$ such that $p_{g}(S)=0$ and neither $\beta$ nor k $\beta$ satisfies Condition (44) of the introduction. If $\beta(\beta-\mathrm{k})<0$, the virtual dimensions of $\left[H_{\beta}\right]^{\mathrm{vir}}$ and $\left[H_{\mathrm{k}-\beta}\right]^{\mathrm{vir}}$ are zero and we use Proposition 2.1 . Assume $\beta(\beta-\mathbf{k}) \geq 0$. By Proposition 3.1 this implies $q(S)=1$ and $\beta(\beta-\mathbf{k})=0$. By Proposition 2.1, the invariants are zero when point insertions are present $(m>0)$. In the case $m=0$, Theorem 1.1 implies

$$
\begin{aligned}
\mathbf{Z}_{\beta}^{P}(S) & =P_{S}^{+}(\beta)\left(q^{1 / 2}+q^{-1 / 2}\right)^{2 \beta^{2}}, \\
\mathbf{Z}_{\mathrm{k}-\beta}^{P}(S) & =P_{S}^{+}(\mathrm{k}-\beta)\left(q^{1 / 2}+q^{-1 / 2}\right)^{2(\mathrm{k}-\beta)^{2}}=P_{S}^{-}(\beta)\left(q^{1 / 2}+q^{-1 / 2}\right)^{2(\mathrm{k}-\beta)^{2}} .
\end{aligned}
$$

The result follows from Dürr-Kabanov-Okonek's wall-crossing formula Theorem 3.2 and a Grothendieck-Riemann-Roch computation giving $s_{1}\left(p_{!} \mathscr{P}\right)=$ $\frac{1}{2}[2 \beta-\mathrm{k}]$.

\section{Appendix A. Reduced Stable PAir invariants}

Recall from Section 2.1 that the natural embedding

$$
\operatorname{Hilb}^{n}\left(\mathcal{C} / H_{\beta}\right) \hookrightarrow S^{[n]} \times H_{\beta}
$$

can be realized as the zero locus of a tautological section of the vector bundle $\mathcal{O}(\mathcal{C})^{[n]}$ on $S^{[n]} \times H_{\beta}$ (see (5) $)$. As we discussed, this induces a relative perfect obstruction theory on $\operatorname{Hilb}^{n}\left(\mathcal{C} / H_{\beta}\right)$. We mentioned how the absolute perfect obstruction theory on $H_{\beta}$ of Dürr-Kabanov-Okonek was used in [14] to construct an absolute perfect obstruction theory on $\operatorname{Hilb}^{n}\left(\mathcal{C} / H_{\beta}\right)$.

The Hilbert scheme $H_{\beta}$ has another perfect obstruction theory also originally discovered by Dürr-Kabanov-Okonek [6]. This perfect obstruction theory comes from embedding $H_{\beta}$ in a compact smooth ambient space as follows. Let $A$ be a sufficiently ample divison 11 and define $\gamma:=[A]+\beta$. Then the AbelJacobi map makes $H_{\gamma}:=\operatorname{Hilb}_{\gamma}(S)$ into a projective bundle over the Picard variety $\operatorname{Pic}^{\gamma}(S)$. In particular, $H_{\gamma}$ is smooth. Consider the closed embedding

$$
H_{\beta} \hookrightarrow H_{\gamma}, C \mapsto A \cup C .
$$

A point $D$ lies in the image of this map if and only if it contains $A$, i.e.

$$
\left.s_{D}\right|_{A}=0 \in H^{0}\left(\mathcal{O}_{A}(D)\right),
$$

where $s_{D}$ denotes the section cutting out $D \subset S$. The family version of this goes as follows. Let $\mathcal{D} \rightarrow H_{\gamma}$ be the universal curve and $\pi: H_{\gamma} \times S \rightarrow H_{\gamma}$ projection. Then the sheaf

$$
F:=\pi_{*}\left(\left.\mathcal{O}(\mathcal{D})\right|_{H_{\gamma} \times A}\right)
$$

\footnotetext{
${ }^{11}$ It suffices to pick $A$ such that $H^{1}(L(A))=H^{2}(L(A))=0$ for all $L \in \operatorname{Pic}^{\beta}(S)$.
} 
has a tautological section with zero locus $H_{\beta}$. Suppose that $\beta$ satisfies the following condition (Condition (i) of Proposition 3.1)

$$
H^{2}(L)=0 \text { for all effective line bundles } L \text { with } c_{1}(L)=\beta \text {. }
$$

Note that this condition is weaker than Condition (44) of the introduction. Then $H^{1}\left(\mathcal{O}_{A}(A+C)\right)=0$ for any $C \in H_{\beta}$. By semicontinuity and base change, $R^{1} \pi_{*}\left(\left.\mathcal{O}(\mathcal{D})\right|_{H_{\gamma} \times A}\right)$ is zero on a Zariski open neighbourhood of $H_{\beta}$. Hence $F$ is a vector bundle on a Zariski open neighbourhood of $H_{\beta}$. This construction gives a perfect obstruction theory on $H_{\beta}$ which we refer to as the reduced perfect obstruction theory (this terminology was not used by DürrKabanov-Okonek). We denote the corresponding virtual cycle by $\left[H_{\beta}\right]^{\text {red }}$. The reduced perfect obstruction theory on $H_{\beta}$ can be combined with the relative perfect obstruction theory on $\operatorname{Hilb}^{n}\left(\mathcal{C} / H_{\beta}\right)$ to give another absolute perfect obstruction theory on $\operatorname{Hilb}^{n}\left(\mathcal{C} / H_{\beta}\right)$. This was carried out in [15, Appendix]. It turns out that the resulting virtual cycle $\left[\operatorname{Hilb}^{n}\left(\mathcal{C} / H_{\beta}\right)\right]^{\text {red }}$ coincides with the one coming from $\mathbb{C}^{*}$-localization of reduced stable pair theory of $X=K_{S}$ to the component $\operatorname{Hilb}^{n}\left(\mathcal{C} / H_{\beta}\right)$ of the $\mathbb{C}^{*}$-fixed locus [15, Appendix]. Note that Condition (24) is automatic when $p_{g}(S)=0$. In this case one can show that $\left[H_{\beta}\right]^{\text {red }}=\left[H_{\beta}\right]^{\text {vir }}$ and $\left[\operatorname{Hilb}^{n}\left(\mathcal{C} / H_{\beta}\right)\right]^{\text {red }}=\left[\operatorname{Hilb}^{n}\left(\mathcal{C} / H_{\beta}\right)\right]^{\text {vir }}$.

If $\beta$ satisfies the stronger condition

$$
H^{2}(L)=0 \text { for all line bundles } L \text { with } c_{1}(L)=\beta,
$$

i.e. Condition (4) of the introduction, then $R^{1} \pi_{*}\left(\left.\mathcal{O}(\mathcal{D})\right|_{H_{\gamma} \times A}\right)=0$ on $H_{\gamma}$ and $F$ is a vector bundle on $H_{\gamma}$. Denote the embedding

$$
\operatorname{Hilb}^{n}\left(\mathcal{C} / H_{\beta}\right) \hookrightarrow S^{[n]} \times H_{\gamma}
$$

by $\iota$. Similarly to (5), define

$$
\mathcal{O}(\mathcal{D}-A)^{[n]}:=\pi_{*}\left(\left.\mathcal{O}\left(S^{[n]} \times \mathcal{D}-S^{[n]} \times A \times H_{\gamma}\right)\right|_{\mathcal{Z} \times H_{\gamma}}\right),
$$

where $\pi: S^{[n]} \times S \times H_{\gamma} \rightarrow S^{[n]} \times H_{\gamma}$ denotes projection. When Condition (44) holds one can compute the virtual cycle as follows [14, Thm. A.7]

$$
\iota_{*}\left[\operatorname{Hilb}^{n}\left(\mathcal{C} / H_{\beta}\right)\right]^{\mathrm{red}}=c_{r}(F) \cdot c_{n}\left(\mathcal{O}(\mathcal{D}-A)^{[n]}\right),
$$

where $r:=\chi(\beta(A))-\chi(\beta)$. Here $\chi(\beta)$ is the holomorphic Euler characteristic of curves in $H_{\beta}$

$$
2 \chi(\beta)=\beta(\beta-\mathbf{k})+2 \chi\left(\mathcal{O}_{S}\right)
$$

and $\chi(\beta(A))$ is defined similarly. The virtual dimension of $\left[\operatorname{Hilb}^{n}\left(\mathcal{C} / H_{\beta}\right)\right]^{\text {red }}$ is

$$
v=\frac{\beta(\beta-\mathbf{k})}{2}+p_{g}(S)+n
$$


which is $p_{g}(S)$ larger than the virtual dimension of $\left[\operatorname{Hilb}^{n}\left(\mathcal{C} / H_{\beta}\right)\right]^{\text {vir }}$.

When only the weaker Condition (24) is satisfied we make the following somewhat surprising observation, which is more or less an immediate corollary of [6. Lem. 2.17].

Proposition A.1. Fix S, $\beta$ such that Condition (24) is satisfied, $H_{\beta} \neq \varnothing$, and $\beta(\beta-\mathbf{k}) \geq 0$. Then $F$ is a vector bundle on $H_{\gamma}$ even though $R^{1} \pi_{*}\left(\left.\mathcal{O}(\mathcal{D})\right|_{H_{\gamma} \times A}\right)$ is in general non-zero. Consequently

$$
\iota_{*}\left[\operatorname{Hilb}^{n}\left(\mathcal{C} / H_{\beta}\right)\right]^{\text {red }}=c_{r}(F) \cdot c_{n}\left(\mathcal{O}(\mathcal{D}-A)^{[n]}\right)
$$

and its virtual dimension is $v=\frac{\beta(\beta-\mathrm{k})}{2}+p_{g}(S)+n$.

Proof. Let $p: \operatorname{Pic}^{\beta}(S) \times S \rightarrow \operatorname{Pic}^{\beta}(S)$ be projection and let $\mathscr{P}$ be a choice of Poincaré bundle on $\operatorname{Pic}^{\beta}(S) \times S$. Let

$$
\mathbb{E}:=\left[E^{0} \stackrel{d^{0}}{\rightarrow} E^{1} \stackrel{d^{1}}{\rightarrow} E^{2}\right]
$$

be a resolution of $R p_{*} \mathscr{P}$ by locally free sheaves. Then Dürr-Kabanov-Okonek found out that ker $d^{1}$ is locally free (Claim). The reason for Claim is the following. If $H_{\mathrm{k}-\beta}=\varnothing$, then $R^{2} p_{*} \mathscr{P}=0$ because $H_{\mathrm{k}-\beta}=\mathbb{P}\left(R^{2} p_{*} \mathscr{P}\right)[6$, Lem. 2.15]. In this case $d^{1}$ is surjective and ker $d^{1}$ is locally free. Suppose $H_{\beta}, H_{\mathrm{k}-\beta}$ are both non-empty. Then we saw in Proposition 3.1 and its proof (i.e. [6, Lem. 2.17] and its proof) that

$$
\begin{aligned}
& \left.R^{2} p_{*} \mathscr{P}\right|_{U}=0,\left.R^{0} p_{*} \mathscr{P}\right|_{V}=0, \\
& \operatorname{Pic}^{\beta}(S)=U \cup V, \operatorname{rk} R p_{*} \mathscr{P}=0,
\end{aligned}
$$

where $U, V$ are the complements of the images of $H_{\mathrm{k}-\beta} \rightarrow \operatorname{Pic}^{\mathrm{k}-\beta}(S) \cong \operatorname{Pic}^{\beta}(S)$, $H_{\beta} \rightarrow \operatorname{Pic}^{\beta}(S)$. We see at once that $R^{1} p_{*} \mathscr{P}$ is torsion. Moreover $\left.R^{1} p_{*} \mathscr{P}\right|_{V}$ is a subsheaf of $E^{1} /\left.\operatorname{im} d^{0}\right|_{V}$. Also $E^{1} /\left.\operatorname{im} d^{0}\right|_{V}$ is locally free because $\left.\left(d^{0}\right)^{*}\right|_{V}$ is surjective. This implies $\left.R^{1} p_{*} \mathscr{P}\right|_{V}$ is zero. Therefore ker $\left.d^{1}\right|_{V}=\left.\operatorname{im} d^{0}\right|_{V}=\left.E^{0}\right|_{V}$ is locally free. Since we already know ker $\left.d^{1}\right|_{U}$ is locally free (because $\left.d^{1}\right|_{U}$ is surjective), this establishes Claim.

Back to the resolution $\mathbb{E}$ of $R p_{*} \mathscr{P}$. Take $\mathbb{E}$ of the following form. Let $\left[E^{1} \stackrel{d^{1}}{\rightarrow}\right.$ $\left.E^{2}\right]$ be a resolution of $R p_{*} \mathscr{P}_{A}(A)$ by locally free sheaves and set $E^{0}:=p_{*} \mathscr{P}(A)$. Note that $p_{*} \mathscr{P}(A)$ is locally free by choice of $A$. We define $\mathbb{E}$ by the following diagram of exact triangles

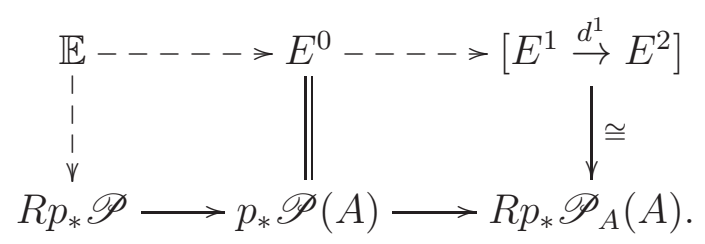


Here $\mathscr{P}_{A}$ is short hand for $\left.\mathscr{P}\right|_{H_{\beta} \times A}$. By Claim, $p_{*} \mathscr{P}_{A}(A) \cong \operatorname{ker} d^{1}$ is locally free. Next, let $\mathcal{Q}$ be a choice of Poincaré bundle on $\operatorname{Pic}^{\gamma}(S)$. The Abel-Jacobi map

$$
\text { AJ }: H_{\gamma}=\mathbb{P}\left(p_{*} \mathcal{Q}\right) \rightarrow \operatorname{Pic}^{\gamma}(S)
$$

is a projective bundle with tautological bundle $\mathcal{O}(1)$. Note that $\mathcal{Q}(1) \cong \mathcal{O}(\mathcal{D})$ on $H_{\gamma} \times S$, therefore (23)

$$
F=\pi_{*} \mathcal{O}\left(\left.\mathcal{D}\right|_{H_{\gamma} \times A}\right) \cong \operatorname{AJ}^{*}\left(p_{*} \mathcal{Q}_{A}\right)(1) .
$$

Since $\operatorname{Pic}^{\beta}(S) \cong \operatorname{Pic}^{\gamma}(S)$ sends $p_{*} \mathscr{P}_{A}(A)$ to $p_{*} \mathcal{Q}_{A}$ we indeed see that $F$ is locally free. Finally the proposition states that $R^{1} \pi_{*}\left(\left.\mathcal{O}(\mathcal{D})\right|_{H_{\gamma} \times A}\right)$ is in general non-zero. This is proved in Remark A.3 below.

If $S, \beta$ satisfies Condition (4), then the invariants $P_{\chi, \beta}^{\text {red }}\left(S, \tau_{0}(\mathrm{pt})^{m}\right)$ are calculated in [15] in the following sense. Via wedging together and integrating over $S$, the classes $\beta, \mathrm{k} \in H^{2}(S, \mathbb{Z})$, and $1 \in H^{4}(S, \mathbb{Z})$ give elements

$$
[\beta],[\mathrm{k}] \in \Lambda^{2} H^{1}(S, \mathbb{Z})^{*} \text {, and }[1] \in \Lambda^{4} H^{1}(S, \mathbb{Z})^{*} .
$$

Wedging together any combination produces an element

$$
\Lambda^{a}[\beta] \wedge \Lambda^{b}[\mathrm{k}] \wedge \Lambda^{c}[1] \in \Lambda^{2 q(S)} H^{1}(S, \mathbb{Z})^{*} \cong \mathbb{Z}, \text { where } a+b+2 c=q(S) .
$$

Here the canonical isomorphism with $\mathbb{Z}$ comes from choosing any integral basis of $H^{1}(S, \mathbb{Z}) \subset H^{1}(S, \mathbb{R})$ compatible with the orientation coming from the complex structure. We then have:

Theorem A.2. [15, Thm. 1.2] Fixing $q, p_{g}, m, n$, there exists a universal function $F_{q, p_{g}, m, n}(\mathbf{x})$ with variables $\mathbf{x}:=\left(x_{1}, x_{2}, x_{3}, x_{4},\left\{x_{a b c}\right\}_{a+b+2 c=q}, t\right)$ such that for any $S$ with $q(S)=q, p_{g}(S)=p_{g}$, and $\beta \in H^{2}(S, \mathbb{Z})$ satisfying Condition (4), $P_{\chi, \beta}^{\mathrm{red}}\left(S, \tau_{0}(\mathrm{pt})^{m}\right)$ is equal to

$$
F_{q, p_{g}, m, n}\left(\beta^{2}, \beta . \mathrm{k}, \mathrm{k}^{2}, c_{2}(S),\left\{\Lambda^{a}[\beta] \wedge \Lambda^{b}[\mathrm{k}] \wedge \Lambda^{c}[1]\right\}_{a+b+2 c=q}, t\right),
$$

where $\chi=1-h+n$ and $2 h-2=\beta(\beta+\mathrm{k})$ is the arithmetic genus of $\beta$.

Remark A.3. Suppose the setting is as in Proposition A.1. We now explain why $R^{1} \pi_{*}\left(\left.\mathcal{O}(\mathcal{D})\right|_{H_{\gamma} \times A}\right)$ is in general non-zero. In Proposition A.1 we show that $F$ is a vector bundle and the reduced virtual cycle is given by (25) when the weaker Condition (24) is satisfied. If $R^{1} \pi_{*}\left(\left.\mathcal{O}(\mathcal{D})\right|_{H_{\gamma} \times A}\right)$ were zero, then the invariants $P_{\chi, \beta}^{\text {red }}\left(S, \tau_{0}(\mathrm{pt})^{m}\right)$ satisfy the same universal formula as Theorem A.2 by the calculation of [15]. However we show by explicit examples in Appendix B that some invariants $P_{\chi, \beta}^{\text {red }}\left(S, \tau_{0}(\mathrm{pt})^{m}\right)$ do not satisfy universality (Examples B.1, B.3, B.5). 


\section{ApPendix B. FAILURE OF Universality: EXAMPLES}

In this appendix we show that Theorem A.2 does not hold for all stable pair invariants $P_{\chi, \beta}^{\text {red }}\left(S, \tau_{0}(\mathrm{pt})^{m}\right), P_{\chi, \beta}\left(S, \tau_{0}(\mathrm{pt})^{m}\right)$. By Main Theorem 1.1 it suffices to prove $P_{S}(\beta)$ is not given by universal functions. We show this on elliptic surfaces with $p_{g}(S)=0$ using calculations of Dürr-Kabanov-Okonek [6].

Let $\pi: S \rightarrow C$ be an elliptic fibration over a curve of genus $g$ [2, Ch. V.6]. We are only interested in the case $S, C$ are algebraic. The generic fibre $F$ is a smooth elliptic curve and we denote by $m_{1} F_{1}, \ldots, m_{r} F_{r}$ the multiple fibres. The canonical divisor is given by

$$
K_{S}=\pi^{*} D+\sum_{i=1}^{r}\left(m_{i}-1\right) F_{i}
$$

for some divisor $D$ of degree $2 g-2+\chi\left(\mathcal{O}_{S}\right)$ on $C$ [2, Cor. 12.3]. In this section, we will make frequent use of logarithmic transformations [2, Ch. V.13]. Given a generic point $x \in C$, a logarithmic transformation replaces the fibre $F$ over $x$ by a multiple $m F, m>1$. The new elliptic fibration $\pi^{\prime}: S^{\prime} \rightarrow C$ has fibre $m F$ over $x \in C$ and the restrictions $\pi^{-1}(C \backslash\{x\}), \pi^{\prime-1}(C \backslash\{x\})$ are biholomorphic as fibre bundles over $C \backslash\{x\}$. One should not think of a logarithmic transformation as a sort of birational transformation. The topology of $S$ can change and $S$ can even become non-algebraic [2, Ch. V.13].

Example B.1. Let $\mathbb{P}^{1} \subset|\mathcal{O}(3)|$ be a generic pencil of cubics on $\mathbb{P}^{2}$ and let $S \rightarrow \mathbb{P}^{1}$ be the universal curve. This is a rational elliptic fibration so $q(S)=$ $p_{g}(S)=0$ and $K_{S}=-F$ (Equation (26) ). We take $\beta=6 \mathrm{k}$. Clearly $\left|6 K_{S}\right|=\varnothing$ so $P_{S}(\beta)=0$. Let $S^{\prime}$ be obtained from $S$ by replacing one general fibre $F$ by a double fibre $2 F_{1}$ and another by a triple fibre $3 F_{2}$. Then $S^{\prime}$ is one of the famous Dolgachev surfaces 12 . The surface $S^{\prime}$ is known to be algebraic satisfying $q\left(S^{\prime}\right)=p_{g}\left(S^{\prime}\right)=0$ and $K_{S^{\prime}}=-F+F_{1}+2 F_{2}$ (Equation (26)). In the Chow group, one has relations $2 F_{1}=3 F_{2}=F$ so $\mathrm{k}^{\prime}=\frac{1}{6}[F]$ in $H^{2}\left(S^{\prime}, \mathbb{Q}\right)$. Taking $\beta^{\prime}=6 \mathrm{k}^{\prime}$, we see that $\left|6 K_{S^{\prime}}\right|=|F| \neq \varnothing$, whereas $\left|K_{S^{\prime}}-6 K_{S^{\prime}}\right|=\left|-5 K_{S^{\prime}}\right|=\varnothing$. Consequently, $P_{S^{\prime}}\left(\mathrm{k}^{\prime}-\beta^{\prime}\right)=0$. Dürr-Kabanov-Okonek's wall-crossing formula (Theorem 3.2) states $P_{S^{\prime}}\left(\beta^{\prime}\right)-P_{S^{\prime}}\left(\mathrm{k}^{\prime}-\beta^{\prime}\right)=1$, so $P_{S^{\prime}}\left(\beta^{\prime}\right)=1$. Since the Chern numbers of $S, \beta$ and $S^{\prime}, \beta^{\prime}$ are the same, this is a counter-example to universality. Note that this does not contradict Theorem A.2. Indeed

$$
H^{2}\left(\mathcal{O}\left(6 K_{S}\right)\right)=H^{0}\left(\mathcal{O}\left(-5 K_{S}\right)\right)=H^{0}(\mathcal{O}(5 F)) \neq 0
$$

so $\beta^{\prime}$ satisfies Condition (44) but $\beta$ only satisfies the weaker Condition (24).

\footnotetext{
${ }^{12}$ The surfaces $S, S^{\prime}$ provide homeomorphic compact simply connected 4-manifolds. S. K. Donaldson famously proved their $C^{\infty}$-structures are different [5]. One can also establish this by showing their Seiberg-Witten invariants are distinct (see [20]).
} 
In order to find more counter-examples to universality, we use the following result [6, Prop. 4.8] (see also R. Friedman and J. W. Morgan [8, Prop. 4.4]).

Proposition B.2 (Dürr-Kabanov-Okonek). Suppose $\beta \in H^{2}(S, \mathbb{Z})$ satisfies $\beta^{2}=\beta .[F]=0$. Then

$$
P_{S}(\beta)=\sum_{\substack{d[F]+\sum_{i} a_{i}\left[F_{i}\right]=\beta \\
d \geq 0,0 \leq a_{i}<m_{i}}}(-1)^{d}\left(\begin{array}{c}
2 g-2+\chi\left(\mathcal{O}_{S}\right) \\
d
\end{array}\right) .
$$

Here we should recall the usual conventions on binomial coefficients. For each $b \geq 0$, define

$$
\left(\begin{array}{l}
a \\
b
\end{array}\right)=\frac{1}{b !} a(a-1) \cdots(a-b+1)
$$

In particular, $\left(\begin{array}{l}a \\ b\end{array}\right)=1$ for $b=0,\left(\begin{array}{l}a \\ b\end{array}\right)=0$ for $0 \leq a<b$, and $\left(\begin{array}{c}-a \\ b\end{array}\right)=(-1)^{b}\left(\begin{array}{c}a+b-1 \\ b\end{array}\right)$.

Example B.3. Let $S$ be an hyper-elliptic surface and $\beta=d[F]$ for any $d \geq 0$. Note that $q(S)=1$ and $p_{g}(S)=0$. Proposition B.2 implies $P_{S}(\beta)=0$ for $d>0$ and $P_{S}(\beta)=1$ for $d=0$. Since $\beta^{2}=\beta . \mathrm{k}=\mathrm{k}^{2}=c_{2}(S)=[\beta]=[\mathrm{k}]=0$ for any $d \geq 0$, this also provides a counter-example to universality. Although $K_{S}$ is a non-trivial torsion element of $A^{1}(S)$, its class $\mathrm{k}=0 \in H^{2}(S, \mathbb{Q})$. The class $\beta=0$ satisfies Condition (24) but not the stronger Condition (44) since $H^{2}\left(\mathcal{O}\left(K_{S}\right)\right) \neq 0$. Hence, there is no contradiction with Theorem A.2.

Finally, we apply Proposition B.2 to a special class of logarithmic transformations discussed in [6, Sect. 4.2]. They will provide more interesting counterexamples to universality. We recall their construction. Fix an elliptic curve $F=\mathbb{C} / \Gamma$ with lattice $\Gamma=\langle 1, \omega\rangle \subset \mathbb{C}$. We apply logarithmic transformations to $\mathbb{P}^{1} \times F \rightarrow \mathbb{P}^{1}$ as follows. Fix a point $t_{1} \in \mathbb{P}^{1}$ and an $m_{1}$-torsion point $\zeta_{1} \in F, m_{1}>0$. The logarithmic transformation $L_{t_{1}}\left(m_{1}, \zeta_{1}\right)\left(\mathbb{P}^{1} \times F\right)$ replaces the fibre over $t_{1}$ by $m_{1} F_{1}$. Continuing in this fashion with other distinct points $t_{2}, \ldots, t_{r} \in \mathbb{P}^{1}$, one obtains a smooth compact complex surface

$$
S:=L_{\underline{t}}(\underline{m}, \underline{\zeta})\left(\mathbb{P}^{1} \times F\right)
$$

which is an elliptic fibration over $\mathbb{P}^{1}$. It has generic fibre $F$ and multiple fibres $m_{1} F_{1}, \ldots, m_{r} F_{r}$. The following proposition [6, Sect. 4.2] summarizes the relevant geometry.

Proposition B.4 (Dürr-Kabanov-Okonek). Suppose $\zeta_{1}, \ldots, \zeta_{r}$ are of the form $\zeta_{i}=\frac{u_{i}+v_{i} \omega}{m_{i}}$ for integers $u_{i}, v_{i}$ satisfying $\operatorname{gcd}\left(m_{i}, u_{i}, v_{i}\right)=1$.

(1) The surface $S$ is projective if and only if $\sum_{i=1}^{r} \zeta_{i}=0$. 
(2) Suppose (1) is satisfied. Then $H^{2}(S, \mathbb{Z}) \cong \mathbb{Z} \oplus G$, where $G$ is the free abelian group generated by $[F],\left[F_{1}\right], \ldots,\left[F_{r}\right]$ modulo the relations

$$
\begin{aligned}
& m_{1}\left[F_{1}\right]=\cdots=m_{r}\left[F_{r}\right]=[F], \\
& u_{1}\left[F_{1}\right]+\cdots+u_{r}\left[F_{r}\right]=0, v_{1}\left[F_{1}\right]+\cdots+v_{r}\left[F_{r}\right]=0 .
\end{aligned}
$$

(3) Suppose (1) is satisfied. Let $\Gamma^{\prime} \subset \mathbb{C}$ be the lattice generated by the elements $1, \omega, \zeta_{1}, \ldots, \zeta_{r}$ and consider the Albanese map Alb $: S \rightarrow \operatorname{Alb}(S)$. Then there exists an isomorphism $\operatorname{Alb}(S) \cong \mathbb{C} / \Gamma^{\prime}$ such that the following diagram commutes

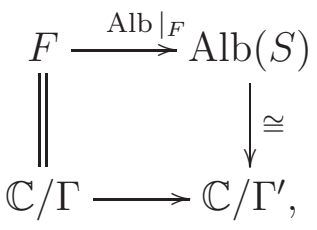

where the bottom map is induced by $\Gamma \subset \Gamma^{\prime}$.

The following example is used in [6, Ex. 4.14] to provide a surface $S$ with $p_{g}(S)=0$ and $P_{S}(\mathrm{k}) \neq 0$. We use it to give an interesting example where universality fails.

Example B.5. Take $\zeta_{1}=\frac{1+\omega}{3}, \zeta_{2}=\frac{1}{3}, \zeta_{3}=\frac{1}{3}$, and $\zeta_{4}=-\frac{3+\omega}{3}$. By Proposition B.4, $S$ is projective, $[F]=3\left[F_{1}\right],\left[F_{4}\right]=\left[F_{1}\right],\left[F_{3}\right]=2\left[F_{1}\right]-\left[F_{2}\right]$, and

$$
\begin{aligned}
H^{2}(S, \mathbb{Z}) & \cong \mathbb{Z} \oplus\left\langle\left[F_{1}\right],\left[F_{2}\right] \mid 3\left[F_{1}\right]=3\left[F_{2}\right]\right\rangle_{\mathbb{Z}} \\
& \cong \mathbb{Z}^{\oplus 2} \oplus \mathbb{Z} / 3 \mathbb{Z} .
\end{aligned}
$$

By Equation (26),$K_{S}=2 F_{1}$ and $\mathrm{k}=\frac{2}{3}[F] \in H^{2}(S, \mathbb{Q})$. We fix $\beta=n\left[F_{1}\right]+\epsilon\left[F_{2}\right]$ with $n \in \mathbb{Z}_{\geq 0}$ and $\epsilon=0,1,2$. The surface $S$ satisfies $q(S)=1$ and $p_{g}(S)=0$. Clearly, $\beta^{2}=\beta \cdot \mathrm{k}=\mathrm{k}^{2}=c_{2}(S)=0$. Let $E$ be the class of the fibre of $S \rightarrow \operatorname{Alb}(S)$. Then Proposition B.4 (3) implies E.F $=9$. Hence $[\beta]=\beta . E=$ $3(n+\epsilon)$. By Proposition B.2

$$
P_{S}(\beta)=\sum_{\substack{\left(3 d+a_{1}+2 a_{3}+a_{4}\right)\left[F_{1}\right]+\left(a_{2}-a_{3}\right)\left[F_{2}\right]=n\left[F_{1}\right]+\epsilon\left[F_{2}\right] \\ d \geq 0, a_{i}=0,1,2}}(d+1) .
$$

For all $(n, \epsilon) \notin\{(0,0),(1,0),(2,0),(0,1)\}$, this is equal to

$$
P_{S}(\beta)=[\beta]-3 \text {. }
$$

For $(n, \epsilon)=(0,0),(1,0),(2,0),(0,1)$, we get the sporadic values

$$
P_{S}(\beta)=1,2,4,1 \text {. }
$$

This gives another counter-example to universality. 
Remark B.6. One can consider reduced stable pair invariants with other insertion classes such as

$$
P_{\chi, \beta}^{\mathrm{red}}\left(S, \tau_{0}(\mathrm{pt})^{m} \tau_{0}\left(\gamma_{1}\right) \ldots \tau_{0}\left(\gamma_{2 q(S)}\right)\right),
$$

where $\gamma_{1}, \ldots, \gamma_{2 q(S)} \in H_{1}(S) /$ torsion is an integral oriented basis [15, Sect. 3]. The $H_{1}$-insertions cut $H_{\beta}$ down to a linear system $|L| \subset H_{\beta}$. Fix any $S, \beta$ with $\beta$ satisfying Condition (24) but not necessarily the stronger Condition (41). Suppose $H_{\beta} \neq \varnothing$ and $\beta(\beta-\mathrm{k}) \geq 0$. Using Proposition A.1, it is easy to see that [15, Sect. 3] continues to hold. Therefore (27) is given by a universal polynomial in $\beta^{2}, \beta . \mathrm{k}, \mathrm{k}^{2}, c_{2}(S)$ exactly as in [15, Thm. 1.1]. Note that this does not contradict Example B.1 where $\left|6 K_{S}\right|=\varnothing$.

Remark B.7. The conditions for the duality formula of Theorem 1.4 are: $p_{g}(S)=0, H_{\beta}, H_{\mathrm{k}-\beta}$ are both non-empty, and $\beta(\beta-\mathrm{k}) \geq 0$. Proposition 3.1 implies $\beta(\beta-\mathrm{k})=0, q(S)=1$, and $S$ is not a ruled surface or a blow-up of a ruled surface. Therefore $S$ is hyper-elliptic, minimal properly elliptic, or a blow-up thereof. Conversely, any hyper-elliptic surface $S$ or blow-up thereof with $\beta=\mathrm{k}$ satisfies the conditions of Theorem 1.4. These examples are boring because $P_{S}^{ \pm}(\mathrm{k})=1$ (by Example B.3 and the blow-up formula [6, Thm. 3.12]). More exciting examples are provided by $S$ as in Proposition B.4 and $\beta=$ k. From Theorem 3.2 it follows that these surfaces generally have $H_{\mathrm{k}} \neq \varnothing$. Blowing up these surfaces, one obtains examples with $H_{\mathrm{k}} \neq \varnothing$ and $\mathrm{k}^{2} \neq 0$.

\section{REFERENCES}

[1] A. Altman, A. Iarrobino, and S. Kleiman, Irreducibility of the compactified Jacobian, Real and complex singularities (Proc. Ninth Nordic Summer School/NAVF Sympos. Math., Oslo, 1976) 1-12 (1977).

[2] W. Barth, C. Peters, and A. van de Ven, Compact complex surfaces, Springer-Verlag (1984).

[3] T. Bridgeland, Hall algebras and curve counting, JAMS 24 969-998 (2011).

[4] H.-l. Chang and Y.-H. Kiem, Poincaré invariants are Seiberg-Witten invariants, Geom. and Topol. 17 1149-1163 (2013).

[5] S. K. Donaldson, Irrationality and the h-cobordism conjecture, J. Diff. Geom. 26 141-168 (1987).

[6] M. Dürr, A. Kabanov, and Ch. Okonek, Poincaré invariants, Topology 46 225-294 (2007).

[7] G. Ellingsrud, L. Göttsche, and M. Lehn, On the cobordism class of the Hilbert scheme of a surface, Jour. Alg. Geom. 10 81-100 (2001).

[8] R. Friedman and J. W. Morgan, Obstruction bundles, semiregularity and Seiberg-Witten invariants, Comm. Anal. Geom. 7 451-495 (1999).

[9] W. Fulton, Intersection theory, Springer-Verlag (1998).

[10] R. Gopakumar and C. Vafa, M-theory and topological strings - I, hep-th/9809187.

[11] R. Gopakumar and C. Vafa, M-theory and topological strings-II, hep-th/9812127. 
[12] T. Graber and R. Pandharipande, Localization of virtual classes, Invent. Math. 135 487-518 (1999).

[13] A. Iarrobino, Punctual Hilbert schemes, Bull. Amer. Math. Soc. 78 819-823 (1972).

[14] M. Kool and R. P. Thomas, Reduced classes and curve counting on surfaces I: theory, Alg. Geom. (Foundation Compos.) 1 334-383 (2014).

[15] M. Kool and R. P. Thomas, Reduced classes and curve counting on surfaces II: calculations, Alg. Geom. (Foundation Compos.) 1 384-399 (2014).

[16] D. Maulik, N. Nekrasov, A. Okounkov, and R. Pandharipande, Gromov-Witten theory and Donaldson-Thomas theory, I, Compos. Math. 142 1263-1285 (2006).

[17] D. Maulik, N. Nekrasov, A. Okounkov, and R. Pandharipande, Gromov-Witten theory and Donaldson-Thomas theory, II, Compos. Math. 142 1286-1304 (2006).

[18] D. Maulik, A. Oblomkov, A. Okounkov, and R. Pandharipande, GromovWitten/Donaldson-Thomas correspondence for toric 3-folds, Invent. Math. 186 435-479 (2011).

[19] D. Maulik, R. Pandharipande and R. P. Thomas, Curves on K3 surfaces and modular forms, J. Topol. 3 937-996 (2010).

[20] J. D. Moore, Lectures on Seiberg-Witten invariants, Lecture Notes in Mathematics 1629, Springer-Verlag (1996).

[21] R. Pandharipande and A. Pixton, Gromov-Witten/Pairs descendent correspondence for toric 3-folds, Geom. and Topol. 18 (2014) 2747-2821.

[22] R. Pandharipande and A. Pixton, Gromov-Witten/Pairs correspondence for the quintic 3-fold, to appear in JAMS (2016), http://dx.doi.org/10.1090/jams/858.

[23] R. Pandharipande and R. P. Thomas, Curve counting via stable pairs in the derived category, Invent. Math. 178 407-447 (2009).

[24] R. Pandharipande and R. P. Thomas, The 3-fold vertex via stable pairs, Geom. and Topol. 13 1835-1876 (2009).

[25] R. Pandharipande and R. P. Thomas, Stable pairs and BPS invariants, J. Amer. Math. Soc. 23 267-297 (2010).

[26] R. Pandharipande and R. P. Thomas, The Katz-Klemm-Vafa conjecture for K3 surfaces, arXiv:1404.6698.

[27] V. Ya. Pidstrigach, Deformations of instanton surfaces, Izv. Akad. Nauk SSSR Ser. Mat. 55 318-338 (1991).

[28] B. Siebert, Virtual fundamental classes, global normal cones and Fulton's canonical classes, in: Frobenius manifolds, ed. K. Hertling and M. Marcolli, Aspects Math. 36 341-358, Vieweg (2004).

[29] C. H. Taubes, The Seiberg-Witten and Gromov invariants, Math. Res. Lett. 2221-238 (1995).

[30] C. H. Taubes, Gr=SW: counting curves and connections, J. Diff. Geom. 52 453-609 (1999).

[31] Y. Toda, Curve counting theories via stable objects I. DT/PT correspondence, J. Amer. Math. Soc. 23 1119-1157 (2010).

[32] E. Witten, Monopoles and four-manifolds, Math. Res. Lett. 1 769-796 (1994).

m.kool1@uu.nl

Mathematical Institute, Utrecht University

Budapestlaan 6, 3584 CD Utrecht, The Netherlands 